\title{
Derivation Without Lexical Rules
}

\author{
Hans-Ulrich Krieger
}

June 1993

Deutsches Forschungszentrum für Künstliche Intelligenz $\mathrm{GmbH}$

Postfach 2080

D-67608 Kaiserslautern, FRG

Tel.: (+49 631) 205-3211/13

Fax: (+49 631) 205-3210
Stuhlsatzenhausweg 3

D-66123 Saarbrücken, FRG

Tel.: (+49 681) 302-5252

Fax: (+49 681) 302-5341 


\section{Deutsches Forschungszentrum für Künstliche Intelligenz}

The German Research Center for Artificial Intelligence (Deutsches Forschungszentrum für Künstliche Intelligenz, DFKI) with sites in Kaiserslautern and Saarbrücken is a non-profit organization which was founded in 1988. The shareholder companies are Atlas Elektronik, Daimler-Benz, Fraunhofer Gesellschaft, GMD, IBM, Insiders, Mannesmann-Kienzle, SEMA Group, Siemens and SiemensNixdorf. Research projects conducted at the DFKI are funded by the German Ministry for Research and Technology, by the shareholder companies, or by other industrial contracts.

The DFKI conducts application-oriented basic research in the field of artificial intelligence and other related subfields of computer science. The overall goal is to construct systems with technical knowledge and common sense which - by using Al methods - implement a problem solution for a selected application area. Currently, there are the following research areas at the DFKI:

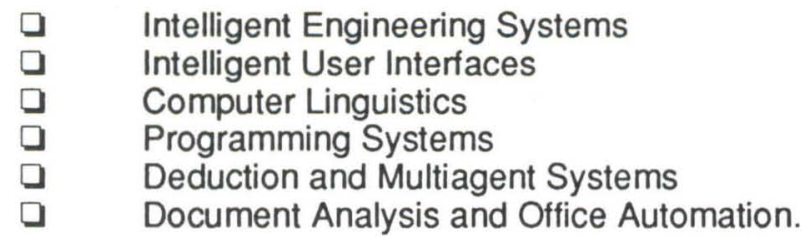

The DFKI strives at making its research results available to the scientific community. There exist many contacts to domestic and foreign research institutions, both in academy and industry. The DFKI hosts technology transfer workshops for shareholders and other interested groups in order to inform about the current state of research.

From its beginning, the DFKI has provided an attractive working environment for Al researchers from Germany and from all over the world. The goal is to have a staff of about 100 researchers at the end of the building-up phase.

Friedrich J. Wendl

Director 
Derivation Without Lexical Rules

\section{Hans-Ulrich Krieger}

DFKI-RR-93-27 
A version of this paper will be published in:

Constraints, Language and Computation, C.J. Rupp, M.A. Rosner \& R.L. Johnson (eds.), Academic Press, 1993, and

IDSIA Working Paper No. 5, Lugano, November 1991.

This work has been supported by a grant from The Federal Ministry for Research and Technology (FKZ ITW-9002 0).

This work may not be copied or reproduced in whole or in part for any commercial purpose. Permission to copy in whole or in part without payment of fee is granted for nonprofit educational and research purposes provided that all such whole or partial copies include the following: a notice that such copying is by permission of Deutsches Forschungszentrum für Künstliche Intelligenz, Kaiserslautern, Federal Republic of Germany; an acknowledgement of the authors and individual contributors to the work; all applicable portions of this copyright notice. Copying, reproducing, or republishing for any other purpose shall require a licence with payment of fee to Deutsches Forschungszentrum für Künstliche Intelligenz. 


\title{
Derivation Without Lexical Rules*

\author{
Hans-Ulrich Krieger \\ krieger@dfki.uni-sb.de \\ German Research Center for Artificial Intelligence (DFKI) \\ Stuhlsatzenhausweg 3 \\ D-6600 Saarbrücken 11, Germany
}

\begin{abstract}
In Krieger and Nerbonne (1992) we showed how to get rid of LEXICAL RULES for DERIVATION, as they are explicated by Pollard and Sag (1987) in HPSG I, Ch. 8.2. We proposed a treatment of derivation NOT by means of traditional lexical rules but instead in terms of PRINCIPLES, RULES, and LEXICAL ENTRIES entirely in the spirit of HPSG, together with unification-based inheritance of a very sophisticated kind. One major disadvantage of this approach was the employment of complex functions in certain principles. In this paper I first extend the old approach and then show how to eliminate these functional dependencies in the domain of derivational morphology by going back to simpler ones like cons, first, and rest. But this simplification is only achieved if we assume more complex feature structures than the ones described in Krieger and Nerbonne (e.g., by introducing two different SUBCAT features) and by proposing modified versions of the old Constituent Order Principle and the Subcategorization Principle for morphology. In addition, I postulate a hierarchy of affixes which is cross-classified, for instance, according to the effects these affixes contribute to the subcategorization information of a compound word.

The structure of the paper is as follows. We start with a very short introduction about the residence of word-formation rules in modern feature-based theories. After that we present our approach to derivational morphology which is distinguished in that it gives up the notion of lexical rule as a single entity (operator). We describe the structure of affixes and words (e.g., which attributes are appropriate?) and introduce the relevant principles and the rule schema of our approach to derivational morphology. The section shows how to reduce functional dependencies to a minimum at the cost of the size of our feature structures. We also present a technique which allows us to state relational dependencies as they are called by HPSG in a functional manner. In the next section we show how the whole treatment works by applying it to tough phenomena from prefixation and suffixation. The section presents many examples, which might serve as a how to guide to a practitioner. After that we explain the idea which will lead us to the affix hierarchy. We will see that the affix hierarchy is inspired by the work of HPSG on structured lexicons (i.e., by the hierarchy of lexical types). A lot of examples will again be given throughout this section. We finish the paper by summarizing our approach and by saying a few words about the topics which we will tackle next.
\end{abstract}

*This work grew out from discussions with John Nerbonne at the DFKI. Thanks are due to Susanne Riehemann and C.J. Rupp for their critique and numerous comments on earlier versions of this paper. The paper has also benefited from numerous people at various workshops where parts of it have been presented, in particular at the ACQUILEX Workshop on Default Inheritance in the Lexicon (Cambridge), the ASL Workshop on DATR (Bielefeld), the Workshop on HPSG and German (Saarbrücken), the Workshop on Constraint Propagation, Linguistic Description, and Computation (Lugano), and the Sprachwissenschaftliches Kolloquium (Univ. of Tübingen). This work was supported by a research grant from the German Bundesministerium für Forschung und Technologie to the DISCO project (FKZ ITW 90020 ). 


\section{Contents}

1 Introduction $\quad 3$

2 The New Approach to Derivational Morphology 4

2.1 The Structure of word and $a f f i x \ldots \ldots \ldots \ldots \ldots$

2.2 Functional vs. Relational Dependencies . . . . . . . . . . . . . . . . . . . . . . . . . . . . . . . . . .

2.3 The Principles and the Rule Schema . . . . . . . . . . . . . . . 10

3 Prefixation and Suffixation 17

3.1 Suffixation . . . . . . . . . . . . . . . . . . . 17

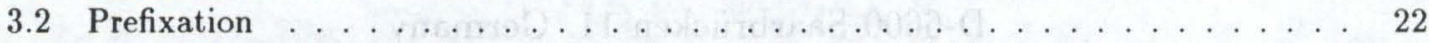

4 The Affix Hierarchy $\quad 25$

4.1 The POS Dimension . . . . . . . . . . . . . . . . . . . . . . . . . . 26

4.2 The CAT Dimension . . . . . . . . . . . . . . . . . . . . . 26

4.3 The SUbCat Dimension . . . . . . . . . . . . . . . . . . . . . . . . . . . 26

4.4 The SEM Dimension . . . . . . . . . . . . . . . . . . . . . . . . . . 28

4.5 The BIND Dimension . . . . . . . . . . . . . . . . . . . . . . . . . . . 28

4.6 More Examples . . . . . . . . . . . . . . . . . . . . . . . 28 


\section{Introduction}

This section treats the question of the RESIDENCE of lexical rules, viz., where does word formation take place - within or without the lexicon? WITHOUT means that the form of lexical rules is different from the structure of lexical entries (lexemes, also possibly morphemes). Although lexical rules in PATR-II [38], D-PATR [24], or in the ACQUILEX-LKB formalism [15] look like ordinary feature structure descriptions-actually they are represented via a collection of path equations using the distinguished attributes (metavariables) IN and OUT - their interpretation however is completely different from that of (normal) feature structure descriptions. The same is true if we move to other theories: f-structures differ in form and interpretation from lexical rules as they are sometimes stated in LFG (cf. the articles in [7]). This observation also holds for the Alvey tools project [36], for the early days of HPSG [20], for the work of Flickinger [19], and for Hoeksema's Categorial Morphology [22]. Lexical rules in HPSG ([34], Ch. 8.2) have the same property, in that feature structure descriptions and lexical rules (form: $\mathrm{AVM}_{1} \longmapsto \mathrm{AVM}_{2}$ ) have nothing in common because they differ in form as well as in interpretation. This remark is supported by the following observation. Feature structures in HPSG are always typed, and these types can be (partially) ordered by means of subsumption. But this isn't true for lexical rules. ${ }^{1}$ A lexical rule as a whole does not have a type and there's no way to relate it to other feature structures. If we assume for the moment that a lexical rule can in principle be typed and resides in the lexicon, this type ought to be a subtype of lexical-sign according to HPSG I and ought to have exactly the three top-level attributes PHON, SYN, and SEM - but this isn't the case, showing our assumption to be false.

By its nature, a lexical rule sets up a relation between two lexemes (actually, between classes of lexemes) - or, in the case of feature-based theories, between two feature structure descriptions. But specifying the exact meaning of this mapping is an open question-nearly all theories have different viewpoints when interpreting lexical rules:

- Are lexical rules functions or perhaps even relations?

- Do they take one argument or arbitrarily many?

- Are they unidirectional or bidirectional?

- Will they be interpreted declaratively $\left(\mathrm{AVM}_{1}\right.$ implies a corresponding $\left.\mathrm{AVM}_{2}\right)$ or procedurally (lexical rule as an instruction to build $\mathrm{AVM}_{2}$ out of $\mathrm{AVM}_{1}$ )?

Lexical rules in HPSG [34] for instance seem to be unary, unidirectional functions. A consequence of this approach to derivation is that every affix (prefixes as well as suffixes) introduces an additional lexical rule to the pool of existing lexical rules. In contrast to the HPSG treatment of derivation, Hoeksema's Categorial Morphology [22] only has a single (binary) rule for prefixation and a single one for suffixation. But in principle, his rules can take arbitrarily many arguments. We will later see that this approach only consists of a single rule (schema), our closest analogue to a lexical rule.

Derivational rules will be MODELED in our approach as feature structure descriptions, just as lexical entries are. This is aesthetically pleasing and has the further advantage of formal clarity because a lexical rule can now be described directly by means of the underlying (feature) logic (for instance Smolka's one [40,41] or Carpenter's [11]). Perhaps most importantly, however, the fact that "lexical rules" and lexical entries are of the same formal (data) type allows one to liberate yet another level of linguistic structure from procedural considerations and therefore to interleave morphological and phrasal processing in a way that is otherwise prohibited. Thus a

\footnotetext{
${ }^{1}$ Of course, one can define a type lexical-rule (and also subtypes of this type) with two distinguished attributes III and OUT. In fact, this is done, for instance, in the LKB formalism of Copestake et al. [15]. These types behave like other types with respect to the subsumption ordering. But as the authors noticed (p. 12), "They differ from normal types in that a lexical rule can be applied to a lexical entry to generate a new lexical entry ...", and this is unfamilar to normal lexical types. Another possibility to cope with the type of a lexical rule is by assigning it a functional type (domain $\longmapsto$ range) - therefore the type hierarchy (strictly speaking: the subsumption ordering) must be generalized to a hierarchy of function types (see for instance [9, 10]).
} 
treatment like this one integrates these two stages because the feature structures directly serve as the interface between them. Another nice advantage in contrast to the HPSG treatment of derivation through lexical rules is that we are able to record the morphosyntactic tree structure of a complex compound word in terms of feature structure descriptions, i.e., it is possible to encode the smaller entities (free and bound morphemes), out of which the new word was built, as parts of the whole structure.

It is worth noting that there is no one-to-one correspondence between traditional lexical rules and objects (feature structures) in our approach-there's no distinguished (unique) element in the lexicon for a given lexical rule. Rather, the information represented in a lexical rule is DISTRIBUTED among lexical entries, principles, and a morphological dominance schema, the only analogue to a lexical rule in this approach.

There are only a few other approaches, which are also distinguished by the absence of traditional lexical rules, e.g., Kathol's work on passive in HPSG [25], Krieger and Nerbonne's treatment of inflection [29], and Russell et al.'s approach to inflection and derivation in the ELU system [37]. ${ }^{2}$

\section{The New Approach to Derivational Morphology}

In HPSG I linguistic knowledge about word formation is encoded through a family of lexical rules (see the introductory remarks in [34], p. 209) which are not feature structures, but rather essentially external operators working on feature structures. This unsatisfactory view appears even more questionable given the view of most linguists that form and meaning are much harder to describe for sentences and phrases than for words. If this is the case, one may ask, why does HPSG treat word formation via external lexical rules rather than in a purely feature-based way? Why not formulate RULES and PRINCIPLES for a word grammar similar to those stated by Pollard and Sag in HPSG I for phrasal and sentential grammar? This general idea is the main working assumption of our approach. We think it is a promising task to approach DERIVATION purely in terms of feature structure descriptions - just in the spirit of HPSG. Recall the following equation from [34], p. 147.

$$
\text { English }=P_{1} \wedge \ldots \wedge P_{n+m} \wedge\left(L_{1} \vee \ldots \vee L_{p} \vee R_{1} \vee \ldots \vee R_{q}\right)
$$

This fundamental equation defines an HPSG grammatical theory for phrases and sentences, and we propose a similar methodology to derivation, relying extensively on rules, principles, and (unification-based) inheritance (for an explanation of (1), see Pollard and Sag, [34], p. 147).

Treating DERIVATION (prefixation as well as suffixation) in our approach will lead to complex words consisting of a head daughter AFFIX and a complement daughter wORD (see example (2)) under the label DTRS. The task of the morphological daughters feature is to encode morphological structure, similarly to how HEAD-DTR and COMP-DTRS do this on the phrasal level (cf. [34]). This is in analogy to the HPSG formulation of phrase structure in features, yielding tree structures. ${ }^{3}$

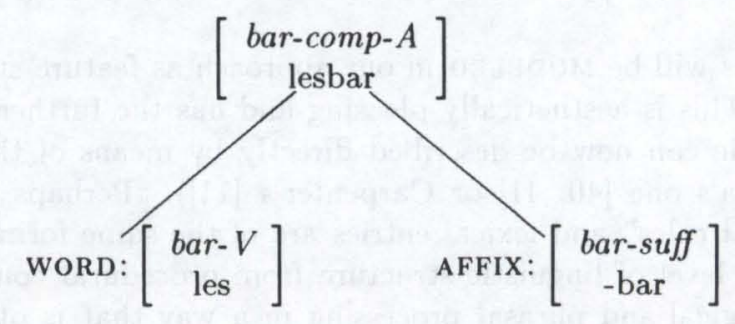

\footnotetext{
${ }^{2}$ ELU treats inflection as well as derivation by means of pure (naive) inheritance. We are convinced however, that this approach is not strong enough for derivation (for an explanation, see below).

${ }^{3}$ Binary trees (possibly combined with unary ones for $\emptyset$ derivation) seem to suffice here for derivation. There are however problematic examples, for instance the German adjective vier+bein+ig (has four legs). At least four possible solutions are possible: (i) use a ternary tree (one head and two complements), (ii) still use a binary tree, therefore violate Aronoff's Word Formation Hypothesis [3] (vierbein and beinig are no legal words), (iii) assume that -beinig is a complex suffix, (iv) write a lexicon entry for vierbeinig.
} 
We include (2) as an example of the hierarchical structure whose analysis is beyond the descriptive reaches of 'naive' inheritance (and of course beyond the power of lexical rules in HPSG which are unable to record such a structure). Here, NAIVE means that a word like for instance the German weg+laufen (run away) is defined by inheriting (unifying) all the properties from the separable prefix weg-and the verb laufen, as well as specifying additional idiosyncratic properties for the new complex lexeme, i.e.,

$$
\text { weglaufen }=[\text { weg }] \wedge[\text { laufen }] \wedge[\ldots \ldots] .
$$

ELU's treatment of derivation (cf. Russell et al. [37], p. 218) is done in such a way, but in general an approach like this one leads to several insurmountable problems:

- If we relied on naive inheritance as the sole descriptive means, it would seem impossible to explain how the iteration of derivational processes could ever lead to different results. If anti(or take the German Vor-or Ur-) is a derivational prefix, and its effect on a stem is described via inheritance, then the effect of inheriting it should be the same, whether there are one, two, or more instances of the same prefix in a word because unification is IDEMPOTENT and our notion of inheritance is defined through unification. Thus a complex word like anti-missile (or Vor+version-pre(liminary) version) would be predicted to be the same as anti-antimissile (or Vortvor+version) ${ }^{4}$ Likewise, such an approach is not capable of explaining the INDIRECT recursion occurring in complex compounds such as institu+tion+al+isa+tion.

- Sole reliance on naive inheritance leaves little opportunity to explain the hierarchical structure often found in morphology, e.g., the difference in bracketing one finds in complex words containing at least two affixes, e.g., [un- $[$ do -able $]]$ as opposed to $[[u n-d o]-a b l e]$. Because inheritance is ASSOCIATIVE and MONOTONIC (in the absence of overwriting), other mechanisms must be at play. Naive inheritance seems incapable of accounting for any structure, let alone ambiguous hierarchical structure.

- Simple examination of derivational results suggests that treating all of them via naive inheritance from a single lexeme will lead to unwieldy lexicons: a form such as German Ableit+bar+keit (derivability) would seem to require that verbal, adjectival, and nominal paradigms be found as heirs of the single lexeme (recall that we dealt with this above by modeling it via mapping from lexeme to lexeme).

- It also turns out that there are technical problems connected with the treatment of derivation as inheritance. These may be summarized, albeit cryptically, in the following way: we should prefer that the result of a category-changing derivational process, e.g., the process which derives derive +able from derive and -able, is a full-fledged member of the target category (of the derivational process) - in this case, the class of adjectives. Now, if the derivational process is modeled by naive inheritance only, then derive+able ought to inherit from the class of verbs (through derive), as well. It is easy to continue this line of reasoning further (consider derive +abil+ity) to see how this sort of explanation leads one to the postulation of lexemes of dubious lineage, inheriting from too many ancestors.

The objections to the description of derivation in terms of naive inheritance do not apply here, since, e.g., tree adjunction is not idempotent-so that, e.g., Vor+version may be distinguished from Vor+vor+version; tree adjunction generates hierarchical structures (evident here), and, as we shall see, it distinguishes inheritance (sharing properties) from the requirements that sublexemes come from particular word classes or types (so that the tree structure for lesbar above cannot be interpreted to mean that the adjective lesbar is in any sense a verb of the same type as lesen or a suffix of the same type as -bar).

\footnotetext{
${ }^{4}$ Permitting iteration of derivational prefixes only to a certain depth (which seems prima facie plausible since, e.g., words such as German Vor+vor+vor+vor+version are questionable), will solve this problem, if every element of the finite set of complex prefixes is coded as a lexical entry. But this attempt of repair is (i) theoretically extremely unsatisfying and (ii) incomplete, because the depth of composition is a subjective measure.
} 


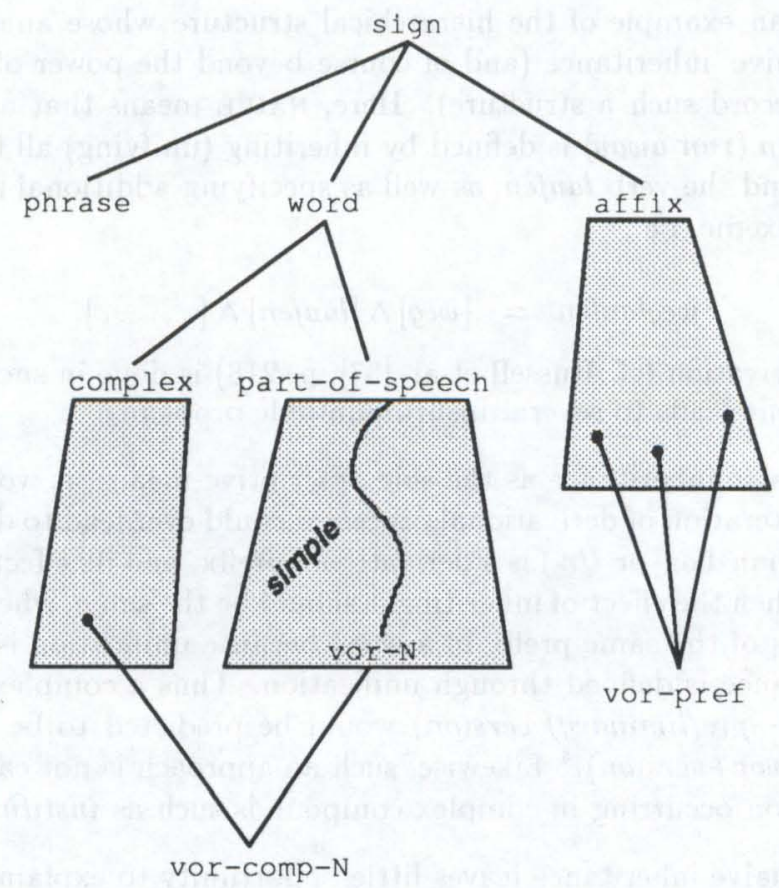

Figure 1: The overall structure of the type lattice for derivation.

Before we're going to apply our approach to derivational morphology and see how the whole treatment works, we must first of all say how the internal structure of a word or an affix looks like, i.e., which attributes are appropriate for a given type and which type is appropriate for a given attribute. In addition, we have to specify the relevant principles and the single rule schema of our approach to derivational morphology.

\subsection{The Structure of word and affix}

Our approach assumes the following top-level attributes for instances of type word (or lexical-sign according to HPSG I; direct subtypes are part-of-speech and complex, cf. Fig. 1):

$$
\left[\begin{array}{l}
\text { word } \\
\text { MORPH word-morphology } \\
\text { SYN word-syntax } \\
\text { SEM word-semantics } \\
\text { CORD list(part-of-speech } \vee \text { affix) } \\
\text { (DTRS affix-word-struct) }
\end{array}\right]
$$

The structure of MORPH resp. the type word-morphology expects certain attributes like STEM, FORM, ENDING, PARADIGM etc. - this is analogous to our old approach presented in [29]. ${ }^{5}$

The value of SEM is in general a predicate-argument structure (see examples). Representing the propositional semantics of words in such a way is along the lines of HPSG and the situation schemata framework (see for instance [18]).

The attribute DTRS is only appropriate for the type complex (and of course for subtypes of this type; cf. Fig. 1). DTRS expects exactly two (typed) attributes, viz., AFFIX and WORD.

\footnotetext{
${ }^{5}$ It might be useful to proclaim an additional attribute SUBCAT under MORPH which is typed to affix. Why so? The next subsection will present TwO alternative subcategorization principles, one (27) having the same formal power as the subcat principle of the old approach, and a stronger one (28) which however assumies a MORPH|SUBCAT attribute in instances of type word. The intention behind the second subcategorization principle is fairly simple-a free word and an affix only come together if they subcategorize each other.
} 


$$
\left[\begin{array}{l}
\text { affix-word-struct } \\
\text { AFFIX affix } \\
\text { WORD part-of-speech }
\end{array}\right]
$$

The structure of word-syntax (SYN) is different to the one given in the old approach with respect to the value of SUBCAT under path SYN|LOC. Instead of proclaiming a subcategorization LIST like HPSG does (type: list(sign)), we propose a different type, called subcat-info, to be appropriate for SYN|LOC|SUBCAT, which must have exactly the four attributes SUBJ (subject), OBJ (object), OBJ2 (second object), and COMPS (the rest of the complements, the oblique objects). ${ }^{6}$

$$
\left[\begin{array}{l}
\text { subcat-info } \\
\text { SUBJ sign } \vee \text { null } \\
\text { OBJ sign } \vee \text { null } \\
\text { OBJ2 sign } \vee \text { null } \\
\text { COMPS list(sign } \vee \text { null) }
\end{array}\right]
$$

This feature structure replaces the well known subcategorization list of HPSG. The motivation for the new structure is primarily a technical one-affixes and the objects they subcategorize are easier to describe and depict if we assume a keyword approach to grammatical relations instead of using a single list (see examples for more details). Note that there are other remarkable HPSGoriented proposals which also give up the original subcategorization list in favour of a keyword approach (see [5], [33], [25]).

The value of the attribute CORD (Constituent oRDer) in (4) is always a non-empty list of feature structures which are either of type part-of-speech or of type affix. This list directly reflects the actual linear surface order of the parts of a complex word (cf. the next subsection to see how CORD works together with the Constituent Order Principle (20)).

Note that, in contrast to CORD, the attribute DTRS only states the hierarchical structure of the new compound but Nот the linear order. Let's take an example to clarify the distinction between CORD and DTRS - the German word Vor+vor+version (cf. the section on Vor prefixation). The analysis tree and the overall structure of Vorvorversion, concerning only the attributes MORPH|FORM, CORD, and DTRS, is depicted in (8) and (7). Don't be confused about the cycle 3 in the feature structure of Version (the explanation follows when we deal with the Constituent Order Principle).

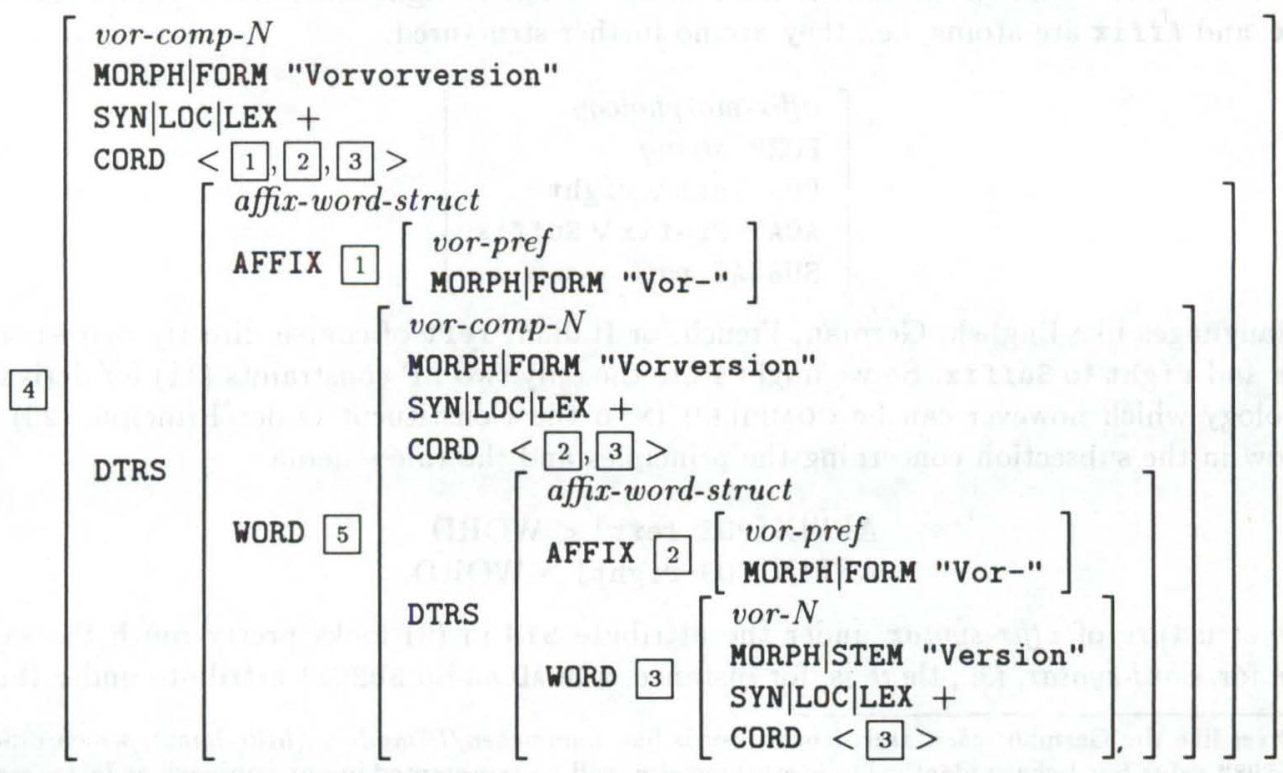

\footnotetext{
${ }^{6}$ Our treatment of subcategorization expects a special type null, whose extension is the unique constant $\mathbf{I l}$. We will use IIL only when we have to state that a grammatical role isn't filled.
} 
(8)

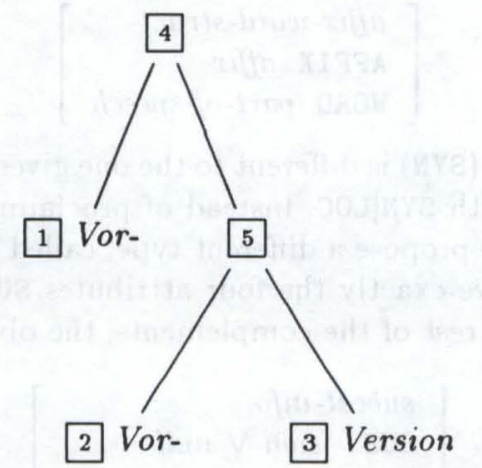

After having inspect the form of words, we must now describe how affixes are internally structured and in what the type word (4) differs from the type affix (9). affix makes use of a new attribute called COND (CONDition; see below), but does neither contain the attribute CORD nor the attribute DTRS. This structural divergence relies on two arguments: (i) affixes do not occur freely and (ii) they are never complex in our approach. Therefore, instances of type affix (cf. Fig. 1) must have exactly the following four attributes:

$$
\left[\begin{array}{l}
\text { affix } \\
\text { MORPH affix-morphology } \\
\text { SYN affix-syntax } \\
\text { SEM affix-semantics } \\
\text { COND }\left(T^{*} \longmapsto\{T, \perp\}\right)
\end{array}\right]
$$

The structure of affix-morphology (10) is different to the one of word-morphology-affixmorphology expects exactly four attributes, viz., FORM, POS, ACAT, and SUBCAT. Although a FORM attribute is assumed, there's neither a STEM nor an ENDING attribute as in word-morphology. ${ }^{7}$ Because of the binary tree property in derivation, the affix takes via its SUBCAT attribute only ONE argument, a free word it subcategorizes for of type part-of-speech. ACAT (Affix CATegory) states whether the affix is classified as a Prefix or as a Suffix. POS (Position) corresponds to the 'position' of the affix on the surface level under the left-to-right order. Note that left, right, Prefix, and Affix are atoms, i.e., they are no further structured.

$$
\left[\begin{array}{l}
\text { affix-morphology } \\
\text { FORM string } \\
\text { POS left } \vee \text { right } \\
\text { ACAT Prefix } \vee \text { Suffix } \\
\text { SUBCAT part-of-speech }
\end{array}\right]
$$

In languages like English, German, French, or Italian, left of course directly corresponds to Prefix and right to Suffix. So we might state the only two LP constraints (11) for derivational morphology which however can be COMPILED INTO the Constituent Order Principle (20) as we will show in the subsection concerning the principles and the rule schema.

$$
\begin{aligned}
& \text { AFFIX [POS left] < WORD } \\
& \text { AFFIX[POS right] > WORD }
\end{aligned}
$$

The structure of affix-syntax under the attribute SYN in (9) looks pretty much the same as the one for word-syntax, i.e., there is, for instance, a HEAD and a SUBCAT attribute under the path

\footnotetext{
${ }^{7}$ Suffixes like the German -chen and -lein in words like Lämmchen/Lämmlein (little lamb), which differ only in their FORH value but behave identical in everything else, will be represented in our approach as instances of the same suffix type but with a different surface realization. However, if we had proposed a STEM attribute for affixes, it would make sense to proclaim a single abstract stem, both for-chen and-lein.
} 
SYN|LOC. This is due to the fact that the Head Feature Principle (24) and the Subcategorization Principle (27) in our approach treat the affix as the HEAD, therefore the SUBCAT and the HEAD values of the affix under path SYN|LOC are coindexed with the same attributes of the mother. Of course, the value for SYN|LOC|SUBCAT is no longer a list-only feature structures of type subcat-info (6) are appropriate (this type is also the appropriate one for SYN|LOC|SUBCAT in word).

The last attribute, COND, defaults to $T$ (this is allowed as the result of the Kleene star in (9)) and is restricted to the FUNCTION TYPE ( $\left.T^{*} \longmapsto\{T, \perp\}\right)$, i.e., the value of cond leads either to a unification failure, because $[\ldots, \operatorname{COND} \perp, \ldots] \doteq \perp$, or leaves the whole structure untouched as a consequence of the fact that $T$ always unifies with everything else. The intention behind COND might sound at the moment a little bit mystical - it is possible to couch relational dependencies purely functionally.

\subsection{Functional vs. Relational Dependencies}

Relational DEPENDENCIES in HPSG can indeed be (re-)formulated functionally. A relational dependency, which is associated with a specific feature structure (type), must hold for the entire feature structure to be a legal one. But if the condition fails, the feature structure isn't licensed by that constraint. Thus, if the unification machinery forces us to state only functional constraints (dependencies) but not relational ones (which is the case in our DISCO project), we must represent relational dependencies in a different way. The technique which we will present here, allows us to embed a restricted relational subset, viz. the set of predicates (in constrast to constructors like cons), in a feature-value logic consisting of function symbols and the propositional connectives $\wedge$, $\vee$, and $\neg$. Note that the class of functions to which COND is typed $\left(T^{*} \longmapsto\{T, \perp\}\right)$, correponds to the class of CHARACTERISTIC FUNCTIONS, known from the theory of computation. To give a flavor how the whole mechanism works, take, for instance, the simplified version of rule 4 (head-adjunct rule) in Pollard and Sag [34], p. 161.

$$
\left[\operatorname{DTRS}\left[\begin{array}{l}
\text { HEAD-DTR } \mid \text { SYN } \mid \text { LOC } \mid \text { HEAD } \mid \text { ADJUNCTS }\{\ldots, 1, \ldots\} \\
\text { ADJ-DTR } \mid \text { SYN } 1
\end{array}\right]\right]
$$

(12) can be rewritten to an equivalent feature structure (13), where the membership of 1 is represented as a functional constraint under attribute COND and member is a function yielding either the value $T$ or $\perp$.

$$
\left[\begin{array}{l}
\text { DTRS }\left[\begin{array}{l}
\text { HEAD-DTR } \mid \text { SYN } \mid \text { LOC } \mid \text { HEAD } \mid \text { ADJUNCTS } 2 \\
\text { ADJ-DTR } \mid \text { SYN } 1
\end{array}\right] \\
\text { COND member }(1,2,2)
\end{array}\right]
$$

Moving back to the structure of affix (9), everything works fine (is monotonic) in all subtypes of the type affix when inheriting the properties of their supertypes because COND should be given the value $T$ if no functional constraint is needed and the COND attributes of affix and all its subtypes must be typed to functions which either yield $T$ or $\perp$ (and of course $T \wedge T \doteq T$ and $T \wedge \perp \doteq \perp$ is the case). ${ }^{8}$

The COND attribute will gain importance when we're going to formulate affix types. We will see that the affix hierarchy consists of types which cannot be described by a 'normal' feature logic without functions or relations (see section on the affix hierarchy). Without functions, we're not able to specify known generalizations about affixes in a proper and convenient way, or in other words, the type definitions turn out to be not specific enough, if we simply omit existing functional dependencies in a feature structure.

The functions under the COND attribute serve as PREDICATES as we have seen above. Besides those predicates we need other functions as well to build up new structures (CONSTRUCTORS) or to access specific elements (ACCESSORS). This approach currently employs only cons, first, and rest in certain affix types and in the Constituent Order Principle. Applying them only

\footnotetext{
${ }^{8}$ In our context, inheriting functions which act as predicates means, to conjoin their results. We expect functions to be RESIDUATED [2, 42] if there's not enough information present to construct the 'full' answer $T$ or $\perp$.
} 
leads to the following nice advantages: (i) the functions are simple in the sense of having a Low COMPUTATIONAL COMPLEXITY - actually cons, first, and rest are at least needed to work on lists, and (ii) the constructor cons is one-to-one and therefore reversible in the sense that there exists an inverse function and not only a relation (in contrast to append), i.e., if we take a list, the first element of this list is uniquely determined, as is the rest. Equation (14) always holds for every list $l$.

$$
\operatorname{cons}(\operatorname{first}(l), \text { rest }(l)) \doteq l
$$

first and rest might be real functions, but they can also be regarded as macro facilities to abbreviate the internal structure of lists. Take for instance the two element list $\langle 1,2\rangle$. This list might be a shorthand for the more elaborated feature structure representation [FIRST 1, REST [FIRST 2, REST NIL]], where FIRST, REST, and NIL are distinguished symbols. Therefore, the following two feature structures are equivalent in terms of the above remark:

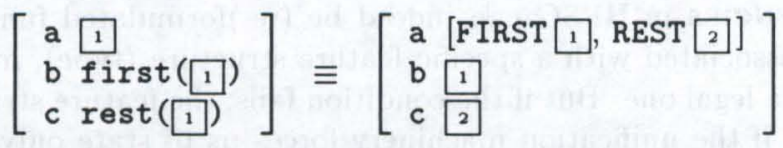

The same argumentation holds for the constructor cons which we will use in (20) - cons too can be seen as a macro. Take for instance the following two equivalent feature structures:

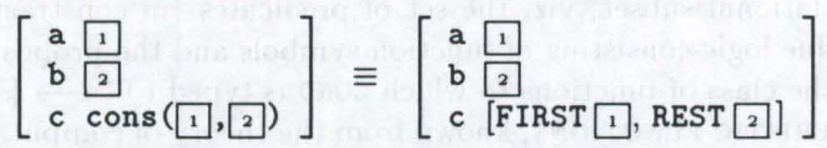

Our approach and the examples to come expect that the following equations always hold for first, rest, and NIL. Note that NIL is overloaded with respect to its type, i.e., NIL denotes the empty list $<>$ as well as a special symbol.

$$
\begin{aligned}
& e q(<>, N I L) \doteq \mathrm{T} \\
& \text { first }(<>) \doteq N I L, \\
& \text { rest }(<>) \doteq N I L .
\end{aligned}
$$

\subsection{The Principles and the Rule Schema}

After we have given an overview about the general structure of affixes and words, it's now the right time to present the principles and the single rule schema. In analogy to the rules stated in HPSG I, [34], Ch. 6, we postulate a very general immediate dominance schema $M A W R$ (Morphological Affix-Word Rule) which is responsible for licensing complex words (see the feature structure (7) of Vorvorversion as a good example for a multiple application of (18))

$$
M A W R=\left[\begin{array}{l}
\text { complex } \\
\text { SYN|LOC|LEX }+ \\
\text { DTRS }\left[\begin{array}{l}
\text { affix-word-struct } \\
\text { AFFIX affix } \\
\text { WORD part-of-speech }
\end{array}\right]
\end{array}\right]
$$

or informally as an ID rule

$$
\mathrm{M}[\mathrm{LEX}+] \longrightarrow \mathrm{A}, \mathrm{W} \text {. }
$$

Note that the value of SYN|LOC|LEX is +, i.e., the whole structure is classified as a (legal) word. This is necessary because LEX is NOT a HEAD feature and will therefore not be percolated via the Head Feature Principle (24) to the mother. Note too that DTRS|WORD|SYN|LOC|LEX is also + as the result of typing DTRS|WORD to part-of-speech. 
Just as HPSG proclaim universal as well as language-specific principles, we will now define and motivate FIVE MORPHOLOGICAL PRINCIPLES, which are consistent with our linguistic data and specified as typed implications: (i) a morphological CONSTITUENT ORDER PRINCIPLE MCOP, (ii) a morphological SURFACE REALIZATION PRINCIPLE MSRP, (iii) a morphological HEAD FEATURE PRINCIPLE MHFP, (iv) a morphological SEMANTICS PRINCIPLE MSP, and (v) a morphological SUBCATEGORIZATION PRINCIPLE MSCP.

Our old Constituent Order Principle (cf. [29]) employed a functional dependency to determine the MORPH|FORM value of the new complex word. ${ }^{9}$ One of our main goals in this paper, however, is to reduce the NUMBER and COMPLEXITY of functional dependencies to a minimum, making the INHERENT KNOWLEDGE of such constraints explicit in feature structure descriptions and using functions only if the problem we want to describe can no longer be stated in a feature logic without functional constraints. Encoding linguistic knowledge in this way is at least interesting from three different viewpoints:

- We have to make our knowledge explicit in a high level language (feature logic) and cannot rely on 'low level' functions (maybe written in Lisp). We cannot longer say OK, here's a function which does the job for me - this was the case in our old approach when we used the function order-constituents, but didn't say how order-constituents really works.

- The whole unification machinery becomes much slender because it is set free from handling functional constraints, at least from complex ones.

- Leaving out functional constraints or restricting oneself to reversible functions becomes important in the light of reversibility - only ONE grammar is needed, both for parsing and generation.

In contrast to the constituent order principle of HPSG which must 'only' capture the linear precedence of the parts of a sentence, the old Constituent Order Principle however was concerned with two jobs-MORPHOTACTICS and MORPHOPHONEMICS (allomorphy). ${ }^{10}$ We will now show how to split the functionality of the old principle into two new principles.

MCOP $(20)$ does the LINEARIZATION part of the old principle, i.e., it determines in what order words and affixes occur on the surface level of the new compound. This (ordered) sequence is then stored under the attribute CORD (see above). But $M C O P$ only applies the very simple function cons in contrast to order-constituents, stated before.

MSRP (23) is concerned with the SURFACE REALIZATION of the new word. The actual realization of the word is assigned to the attribute MORPH|FORM. Of course, MSRP applies the function realize-surface, but this function is set free from the whole morphotactics and handles 'only' the morphophonemics. It. MIGHT directly correspond to a call of a two-level morphological component [26], i.e., as a foreign function interface to a system outside of the apparatus of feature logic. We will have nothing to say about this here, although we are convinced that allomorphy can be handled entirely in a feature unification-based framework (see for instance the novel work of Bird on finite-state phonology in HPSG [4] and Krieger et al. [30] on feature-based allomorphy, showing for the first time how to encode and process two-level automata within typed feature formalisms; cf. [27] for the general framework). ${ }^{11}$

\footnotetext{
${ }^{9}$ The form of the old Constituent Order Principle was mainly inspired by HPSG ([34], p. 169):

$\left[\begin{array}{l}\text { complex } \\ \text { DTRS affix-word-struct }\end{array}\right] \Rightarrow\left[\begin{array}{l}\text { complex } \\ \text { MORPH|FORM order-constituents ( } 1 \\ \text { DTRS } 1\end{array}\right]$

${ }^{10}$ Linear precedence in HPSG is given by a set of linear precedence (LP) constraints (actually, there are two kinds of LP statements, viz., $X<Y$ and $X \ll Y$; for a detailed explanation, see [34], Ch. 7). This sort of linear precedence roughly corresponds to morphotactics, and in fact, we have given above the only two LP constraints for derivational morphology (11) which we will compile into the new principle and the feature structure descriptions for affixes. However, there is no pendant to morphophonemics on the sentence level (except perhaps the behaviour of clitics).

${ }^{11}$ The general rule of thumb for derivation in German is to concatenate the affix and the stem or the former constructed complex word in case of multiple-affix application. However, there are many exceptions to this rule, for example ess + bar $\rightarrow$ eßbar (edible) or entschuldig + bar $\rightarrow$ entschuldbar (excusable).
} 
A treatment of such a kind allows us to reduce the complexity originally expressed in the old constituent order principle by dividing the whole work into two parts. This is only possible because we make a clear distinction between morphotactics and morphophonemics. Note that MAWR (18) and MCOP (20) are responsible for the WHOLE morphotactics.

$$
M C O P=\left\{\begin{array}{l}
{\left[\begin{array}{l}
\text { complex } \\
\text { DTRS affix-word-struct }
\end{array}\right] \Rightarrow} \\
{\left[\begin{array}{l}
\text { complex } \\
\text { DTRS }\left[\begin{array}{l}
\text { AFFIX } 1][\text { MORPH } \mid \text { POS } 3] \\
\text { WORD } \mid \text { CORD } 2
\end{array}\right]
\end{array}\right]}
\end{array}\right.
$$

The 3-place cons in $M C O P$ is a generalized version of the binary function cons - the third argument (the value of POS) determines whether the first argument will be the first ([POS left]) or the last element ([POS right]) of the new list. Another possibility to do things right is to use a binary, polymorphical version of cons which determines with respect to the type of its first argument (type of the value of the the AFFIX attribute) whether to cons left or right-in this case, the attribute POS can be omitted.

$$
\begin{aligned}
& \text { cons }(\text { first }, \text { rest }, \text { left })=\langle\text { first } \text {.rest }\rangle \text { vs. } \\
& \text { cons }([\text { prefix }], \text { rest })=<[\text { prefix }] . \text { rest }\rangle \\
& \text { cons }(\text { first }, \text { rest }, \text { right })=\left\langle\text { first } . \text { rest }^{-1}\right\rangle^{-1} \text { vs. } \\
& \text { cons } \left.([\text { suffix }], \text { rest })=<[\text { suffix }] . \text { rest }^{-1}\right\rangle^{-1}
\end{aligned}
$$

The incorporation of the two LP constraints (11) via cons into the feature structure description of $M C O P(20)$ has the nice advantage that neither additional principles nor new rule schemata must be introduced. From a processing point of view, this compilation is also interesting because we are no longer forced to say when to evaluate the LP constraints for morphology.

This version of the Morphological Constituent Order Principle MCOP assumes that the CORD attribute is also appropriate for simple words (words having no internal constituent structure, i.e. no attribute DTRS) and not only for complex ones. ${ }^{12}$ This is due to the fact that the second argument of cons in MCOP (20) has to be a LIST and this argument is coindexed with the value of DTRS|WORD|CORD via 2 2 , which might be a simple word. In the case of simple words, the value of CORD is a list containing the WHOLE feature structure ITSELF as the only element! Notice that a feature structure like (22) is forbidden according to HPSG I, p. 37.

$$
1\left[\begin{array}{l}
\text { vor- } N \\
\text { MORPH|STEM "Version" } \\
\ldots \ldots \\
\text { CORD }<1>1>
\end{array}\right]
$$

A more general example, viz. the analysis tree (8) and the feature structure (7) of Vorvorversion, was given in the previous subsection. With assistance of the new constituent order principle and the assumption that simple words refer to themselves via CORD, we can now verify that (7) depicts indeed the legal structure for Vorvorversion. Because Vor-is classified as a prefix, we know that the value of its POS attribute must be left, i.e., the cons in MCOP is enforced to cons Vor- left and this new sequence is then stored under the CORD attribute of the mother.

However, we're Nот directly concerned with the morphological surface realization of the new compound - that's the main reason why we propose the functional dependency realize-surface in the Surface Realization Principle MSRP (23). Because MSRP is liberated from the whole morphotactics, realize-surface is in fact less complex than the function order-constituents of the old constituent order principle.

\footnotetext{
${ }^{12}$ Using TYPE NEGATION, one can easily define a type simple abbreviating a certain sublattice in Fig. 1 which contains only simple words (note the marked region in Fig. 1): simple $:=$ part-of-speech $\wedge \neg$ complex.
} 


$$
M S R P=\left\{\begin{array}{l}
{\left[\begin{array}{l}
\text { complex } \\
\text { DTRS affix-word-struct }
\end{array}\right] \Rightarrow} \\
{\left[\begin{array}{l}
\text { complex } \\
\text { MORPH|FORM realize-surface }(1,, 2) \\
\text { CORD } 1 \\
\text { DTRS } 2
\end{array}\right]}
\end{array}\right.
$$

Likewise for derivation, the formulation of the Morphological Head Feature Principle was taken over from HPSG ([34], p. 58) - only certain attributes and type names were altered. Among other things, MHFP is responsible for deducing the category of a new word from the category of the head daughter.

$$
\text { MHFP }=\left\{\begin{array}{l}
{\left[\begin{array}{l}
\text { complex } \\
\text { DTRS affix-word-struct }
\end{array}\right] \Longrightarrow} \\
{\left[\begin{array}{l}
\text { complex } \\
\text { SYN } \mid \text { LOC } \mid \text { HEAD } 1 \\
\text { DTRS } \mid \text { AFFIX } \mid \text { SYN } \mid \text { LOC } \mid \text { HEAD } 1
\end{array}\right]}
\end{array}\right.
$$

The Morphological Semantics Principle corresponds to the simplest version of HPSG I ([34], p. 99): the semantics of the mother is equal to the semantics of the head daughter.

$$
M S P=\left\{\begin{array}{l}
{\left[\begin{array}{l}
\text { complex } \\
\text { DTRS affix-word-struct }
\end{array}\right] \Rightarrow} \\
{\left[\begin{array}{l}
\text { complex } \\
\text { SEM } 1 \\
\text { DTRS } \mid \text { AFFIX } \mid \text { SEM } 1
\end{array}\right]}
\end{array}\right.
$$

In order to eliminate the complex function construct-subcat in the subcategorization principle of the old approach [29], we make a distinction between MORPHOLOGICAL and SYNTACTIC subcategorization (see example (26)): an affix looks for the right feature structure to bind (morphological subcategorization), but the subcategorization information of the new complex word (its sentential subcategorization) directly comes from the syntactic subcategorization of the affix by means of structure sharing. ${ }^{13}$ We will see that the new Morphological Subcategorization Principle $M S C P$ makes no reference. to a function any longer-we use equality (structure sharing) only. To make this approach a bit more concrete, we take a look on the almost regular suffix -bar (cf. the subsection on bar suffixation) and then move straight on to the new Subcategorization Principle.

Feature structure (26) classifies -bar as a suffix via the ACAT (resp. POS) attribute, but syntactically -bar must be regarded, as a consequence of the head feature principle, as an adjective (because of [SYN|LOC|HEAD|MAJ A]). Note that it subcategorizes for a bar verb via MORPH|SUBCAT. The structure sharing between attributes under MORPH|SUBCAT|SYN|LOC|SUBCAT and SYN|LOC|SUBCAT guarantees that MSCP transports the right subcat information (the one under SYN|LOC|SUBCAT) from the head (the affix) to the mother. Note too that we are sometimes forced to state that the value of OBJ or OBJ2 is NIL, or to assign the empty list $(<>)$ to COMPS (this can be technically achieved via subtypes of (a more general) subcat-info).

\footnotetext{
${ }^{13}$ The form of the old subcategorization principle for morphology differs from the sentential subcat principle presented by Pollard and Sag in [34], p. 71:

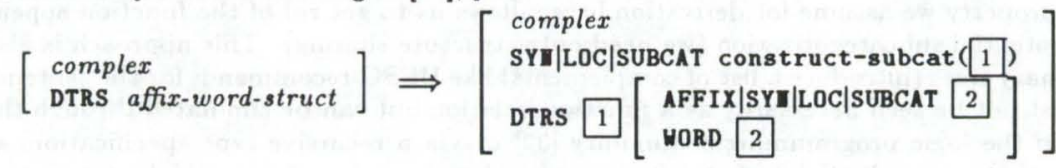




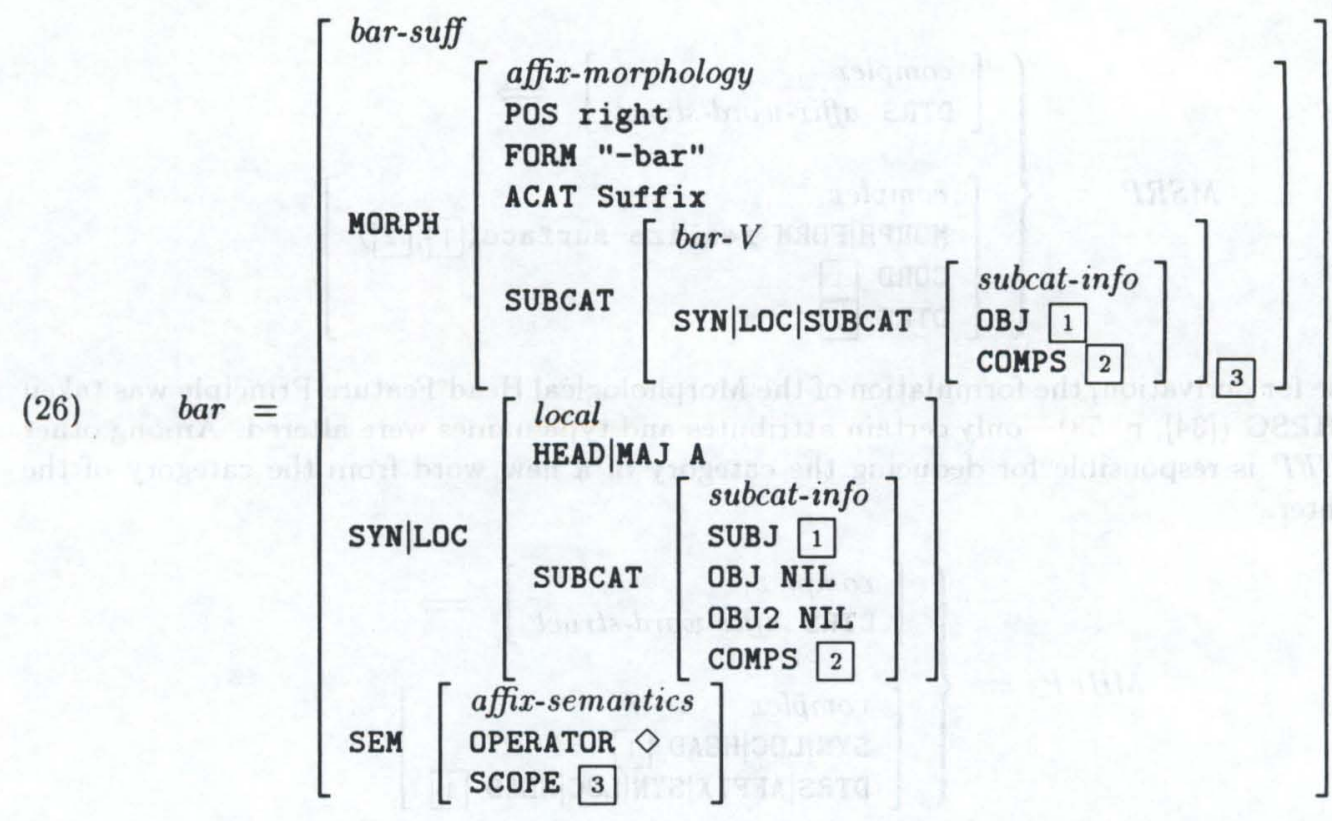

The following Morphological Subcategorization Principle MSCP takes care that the AFFIX will bind the right WORD via DTRS|AFFIX|MORPH|SUBCAT (value: single feature structure of type part-of-speech) and additionally determines the right subcategorization information of the new COMPOUND word through DTRS|AFFIX|SYN|LOC|SUBCAT (typed to subcat-info):

$$
M S C P=\left\{\begin{array}{l}
{\left[\begin{array}{l}
\text { complex } \\
\text { DTRS affix-word-struct }
\end{array}\right] \Longrightarrow} \\
{\left[\begin{array}{l}
\text { complex } \\
\text { SYN|LOC|SUBCAT } 1 \\
\text { DTRS }\left[\begin{array}{l}
\text { AFFIX } \\
\text { WORD } 2
\end{array}\right]
\end{array}\right]}
\end{array}\right.
$$

The reason for the simplicity of $M S C P$ arises from the new structure of the affixes: the distinction between morphological and syntactic/sentential subcategorization and the complex structure sharing of information between subcat-info attributes under MORPH|SUBCAT|SYN|LOC|SUBCAT and SYN|LOC|SUBCAT guarantees that $M S C P$ works proper. ${ }^{14}$

However, it might be useful to have even a STRONGER version of the Subcategorization Principle (28). Such a stronger principle is often useful to handle subregularities and EXCEPTIONS in word class definitions - it helps to avoid the definition of additional subtypes of a given type or the introduction of non-monotonic extensions to the type system resp. to the unifier. We will study an example in the next section where the more restricted Subcategorization Principle is in fact applied to exceptions occuring in the context of bar suffixation. (28) presumes that the affix AND the word will SUBCATEGORIZE EACH OTHER. As we mentioned above, this version also assumes that MORPH|SUBCAT is appropriate for the type part-of-speech, i.e., a free word (the lexeme) also possesses two SUBCAT attributes.

\footnotetext{
${ }^{14}$ Moreover, the binary tree property we assume for derivation here, allows us to get rid of the function append used by Pollard and Sag for sentential subcategorization (we need only structure-sharing). This approach is also capable of working with non-binary trees (introduce a list of complements) like HPSG recommends for the sentence level, because a list append must not be seen necessarily as a function/relation but can be simulated through the difference list technique used in the logic programming community [32] or via a recursive type specification, as Aït-Kaci has shown in [1] (note that we won't stick to functions or even relations as long as possible).
} 


$$
M S C P^{*}=\left\{\begin{array}{l}
{\left[\begin{array}{l}
\text { complex } \\
\text { DTRS affix-word-struct }
\end{array}\right] \Longrightarrow} \\
{\left[\begin{array}{l}
\text { complex } \\
\text { SYN|LOC|SUBCAT } 1 \\
\text { DTRS }\left[\begin{array}{l}
\text { AFFIX } 3\left[\begin{array}{l}
\text { SYN } \mid \text { LOC } \mid \text { SUBCAT } 1 \\
\text { MORPH } \mid \text { SUBCAT } 2 \\
\text { WORD } 2[\text { MORPH|SUBCAT } 3
\end{array}\right]
\end{array}\right]
\end{array}\right]}
\end{array}\right.
$$

Although Pollard and Sag strictly type the attributes of feature structures in general, they do not explicitly state that PRINCIPLES as well as RULES may also be regarded as types. But we may interpret them as types which have to satisfy the suBSUMPTION relation only. ${ }^{15}$ In taking this step, one has to INTEGRATE them CONSISTENTLY into the subsumption lattice (cf. the types $M H F P_{c}, M S C P_{c}, M S P_{c}, M C O P_{c}$, and $M S R P_{c}$ in Fig. 2).

With respect to equation (1), we extend the set of principles and the set of rules by adding $M C O P, M S R P, M H F P, M S P, M S C P$, and MAWR to them. Finally, in typing the antecedents of the implications, we must take care, since not every principle can be combined with every rule or lexical entry. Because only morphological affix-word structures are examined in this paper, equation (1) allows us to unify the feature structure descriptions associated with (the right-hand sides of) MCOP, MSRP, MHFP, MSP, MSCP, and MAWR (call the result AWREPs), and to regard this feature structure as a RESTRICTION (a constraint, a filter) for all feature structures belonging to this new type. All complex words containing the attribute DTRS must satisfy this type restriction, i.e., must be of the type $A W R E \mathcal{B}$, or equivalently, $A W R E \mathcal{P}$ s states what is common to ALL morphologically complex words.

$$
A W R E P S=M H F P_{c} \wedge M S C P_{c} \wedge M S P_{c} \wedge M C O P_{c} \wedge M S R P_{c} \wedge M A W R
$$

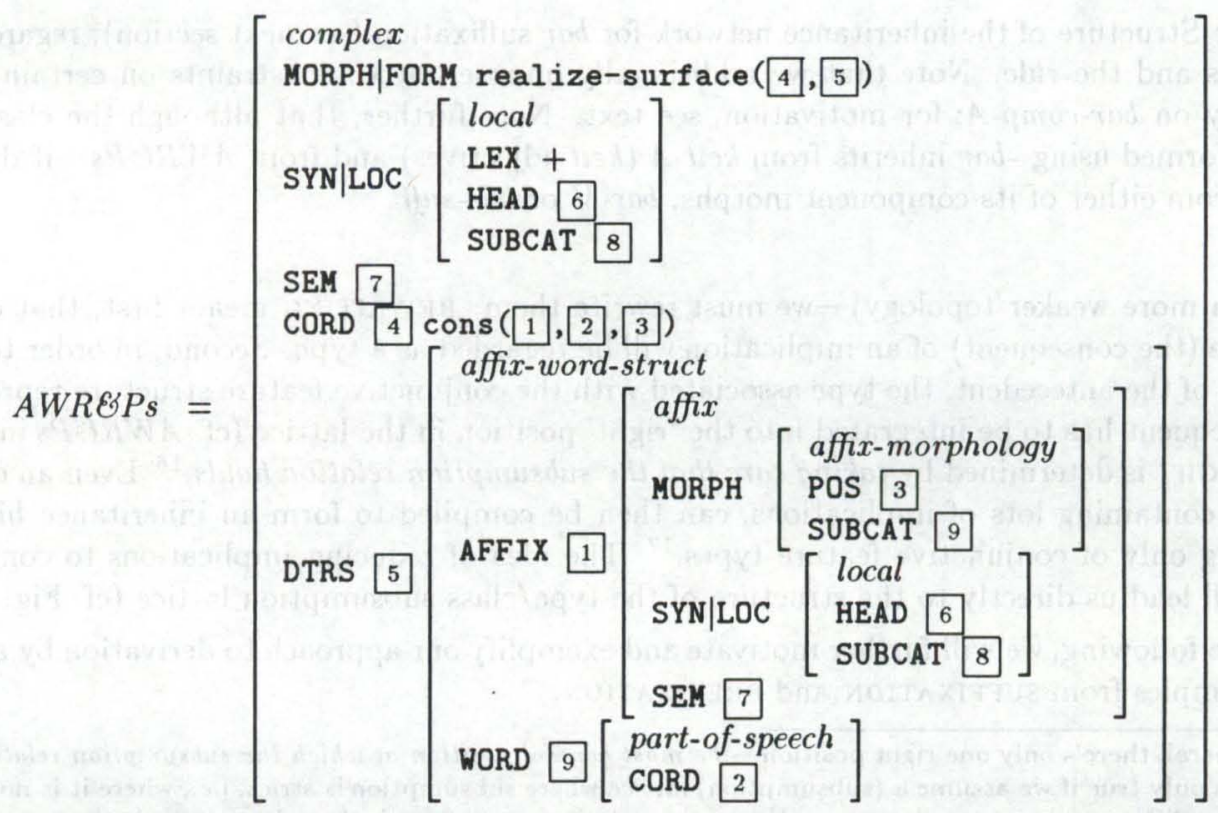

Trying to encode rules and principles explicitly as elements of a type subsumption lattice (inheritance network) along the lines of HPSG ([34], Ch. 8), requires a 'rewriting' step. Because of their implicative nature, we cannot state principles DIRECTLY as types in a distributive lattice (or

\footnotetext{
${ }^{15}$ Principles CONSTRAIN existing types and therefore must be interpreted as supertypes. In translating a principle - usually expressed as a conditional-into a type, we only use the the right side of the conditional, the consequent (for a motivation, see below). Note the similarity between principles here and completion rules in the Alvey tools project [36].
} 


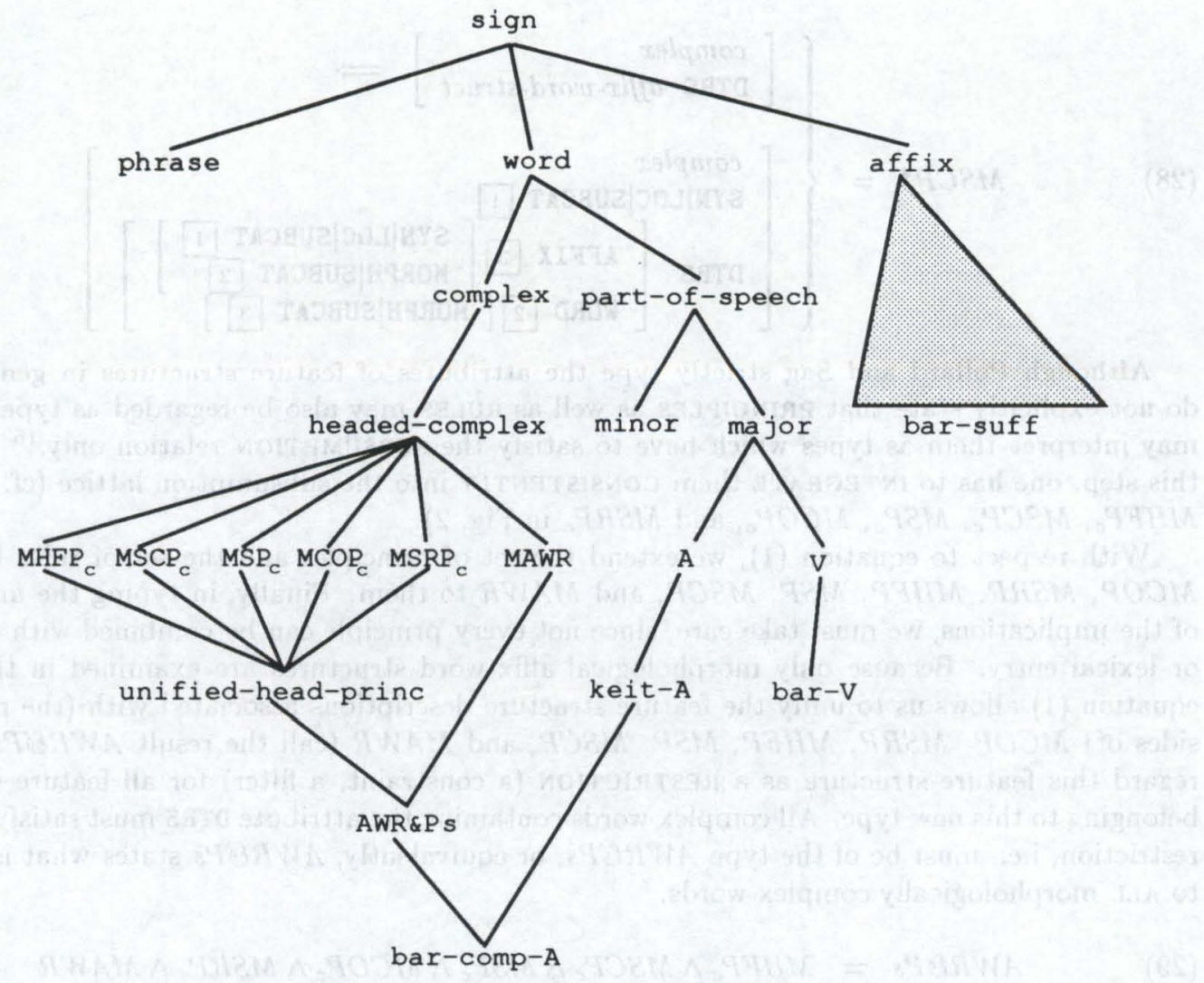

Figure 2: Structure of the inheritance network for bar suffixation (see next section), regarding the principles and the rule. Note that we additionally impose LOCAL constraints on certain classes, especially on bar-comp- $A$; for motivation, see text. Note further, that although the class of adjectives formed using -bar inherits from keit-A (keit adjectives) and from $A W R E P s$-it does NOT inherit from either of its component morphs, bar- $V$ or bar-suff.

even in a more weaker topology) - we must rewrite them. REWRITING means first, that only the right side (the consequent) of an implication will be regarded as a type. Second, in order to obtain the force of the antecedent, the type associated with the conjunctive feature structure representing the consequent has to be integrated into the 'right' position in the lattice (cf. AWREPs in Fig. 2), where RIGHT is determined by taking care that the subsumption relation holds ${ }^{16}$ Even an equation like (1), containing lots of implications, can then be compiled to form an inheritance hierarchy, consisting only of conjunctive feature types. ${ }^{17}$ The idea of reducing implications to conjunctive types will lead us directly to the structure of the type/class subsumption lattice (cf. Fig. 2).

In the following, we will further motivate and exemplify our approach to derivation by applying it to examples from SUFFIXATION and PREFIXATION.

\footnotetext{
${ }^{16}$ In general, there's only one right position-the most general position at which the subsumption relation holds. But this is only true if we assume a (subsumption) lattice where subsumption is strict, i.e., where it is not possible to have two different types standing in a subsumption relation, even though their denotation is the same.

${ }^{17}$ The rewriting step is subtle in that it moves information from object-language implicational statements into restrictions in the type hierarchy which forms the skeleton of the interpretation. On the one hand, because of general laws of interpretation for feature logics, we have the following inference for the feature structures Ante and Conseq: from the principle Ante $\Rightarrow$ Conseq, we know that Ante $\sqsubseteq$ Conseq. On the other hand, the principles always add information to a feature structure description to which they are applied, so that Ante always subsumes Conseq, i.e., Ante $\sqsupseteq$ Conseq. This leads to an effective identification of Ante and Conseq which is realized in the type hierarchy.
} 


\section{Prefixation and Suffixation}

This section is intended to show how the principles, the single rule schema, and the lexical entries fit together by applying them to concrete examples. We show that the approach presented up to here is capable of handling really tough phenomena from the domain of derivational morphology. In addition, we argue that subregularities and exceptions to a certain degree can be handled by the stronger Subcategorization Principle $M S C P^{*}(28)$. In general, capturing such non-regular data through a non-monotonic device might be the better way. ${ }^{18}$ The non-monotonic mechanism we would like to employ during this section is termed SINGLE LINK OVERWRITING (SLO). ${ }^{19}$

\subsection{Suffixation}

The treatment of German bar suffixation is interesting from different points of view and presents severe problems, which can however be adequately solved in our approach. ${ }^{20}$

- sporadic applicability -bar suffixes many verbs but not all,

- partial regularity many bar derivatives have regular forms but irregular semantics or irregular forms with regular syntax and semantics,

- category change bar suffixation changes (syntactic) category (TV $\sim A$ ),

- subcategorization change the subcategorization information of $\mathrm{V}+b a r$ changes, the semantic argument positions in the scope of -bar, on the other hand, do not change.

Starting with a verb like the German lesen (to read), where bar suffixation is perfectly regular, we may construct a possible lexicon entry with respect to the inheritance network of Fig. 2 .

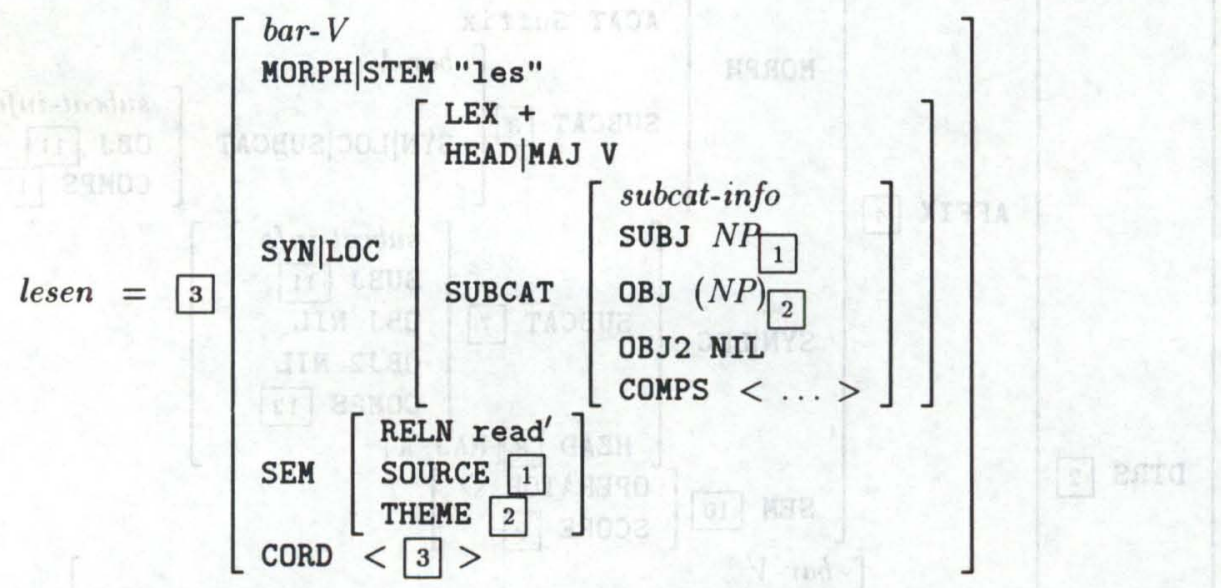

Notice that although lesen is syntactically classified as a verb (V), it is an instance of the class bar- $V$ (verbs that may combine with -bar). Note also that we employ here the LExEME lesen rather than, e.g., the infinitive in lesen's paradigm - this is compatible with the fact that only the stem les- is found in the derived word.

Moving now to -bar, we regard -bar (see (26)) as the HEAD of the morphological complex with category adjective (A); it may function as a head even though it fails to appear as a FREE

\footnotetext{
${ }^{18}$ This paper neither discusses the pros and cons of non-monotonic extensions to the predominant monotonic unification paradigm of computational linguistics nor given proposals so far (see for instance [6], [8], [12], [14], [16], [17], [19], [21], [23], [37], [39]).

${ }^{19}$ It is shown in [28] that SLO is interesting from different points of view, viz., (i) it is capable of expressing the relevant linguistic facts in a natural way, (ii) it is efficiently implementable, (iii) it has nice theoretical properties, and (iv) it is a very conservative extension to the standard monotonic unification machinery.

${ }^{20}$ Riehemann [35] adopt the approach to German bar adjectives, using the Mannheim Zeitungskorpus as a basis. In addition, she poses several questions concerning the redundancy of the basic proposal, for instance that word syntax stated through (18) is not needed.
} 
word. Instead, it occurs only as a Bound morpheme (instance of the class bar-suff; cf. Fig. 2). As a result of the Head Feature Principle the mother automatically obtains the category of the head daughter-and this is exactly what we want, since les+bar (readable) is an adjective. The Affix-Word Rule, the Subcategorization Principle and the specification of MORPH|SUBCAT to be an (underspecified) instance of type $b a r-V$ additionally guarantee that - bar only combines with bar verbs. Semantically, -bar functions as a modal operator, working on the propositional content of lesen (read) to create a proposition, asserting the possibility of reading. We note here the co-specification between the semantics of the subcategorized element under MORPH|SUBCAT and the value of the SCOPE attribute in the modal proposition. These assumptions has led us to the structure of -bar which is depicted in (26). The entries for lesen and -bar together with the Affix-Word Rule and the Morphological Principles permit us therefore to construct a well-formed feature structure for les+bar, and also to reject ill-formed feature structures, so that we can show that (32) is the predicted structure and (2) the corresponding analysis tree. This meshing of mechanisms ensures that lesbar has the right internal structure.

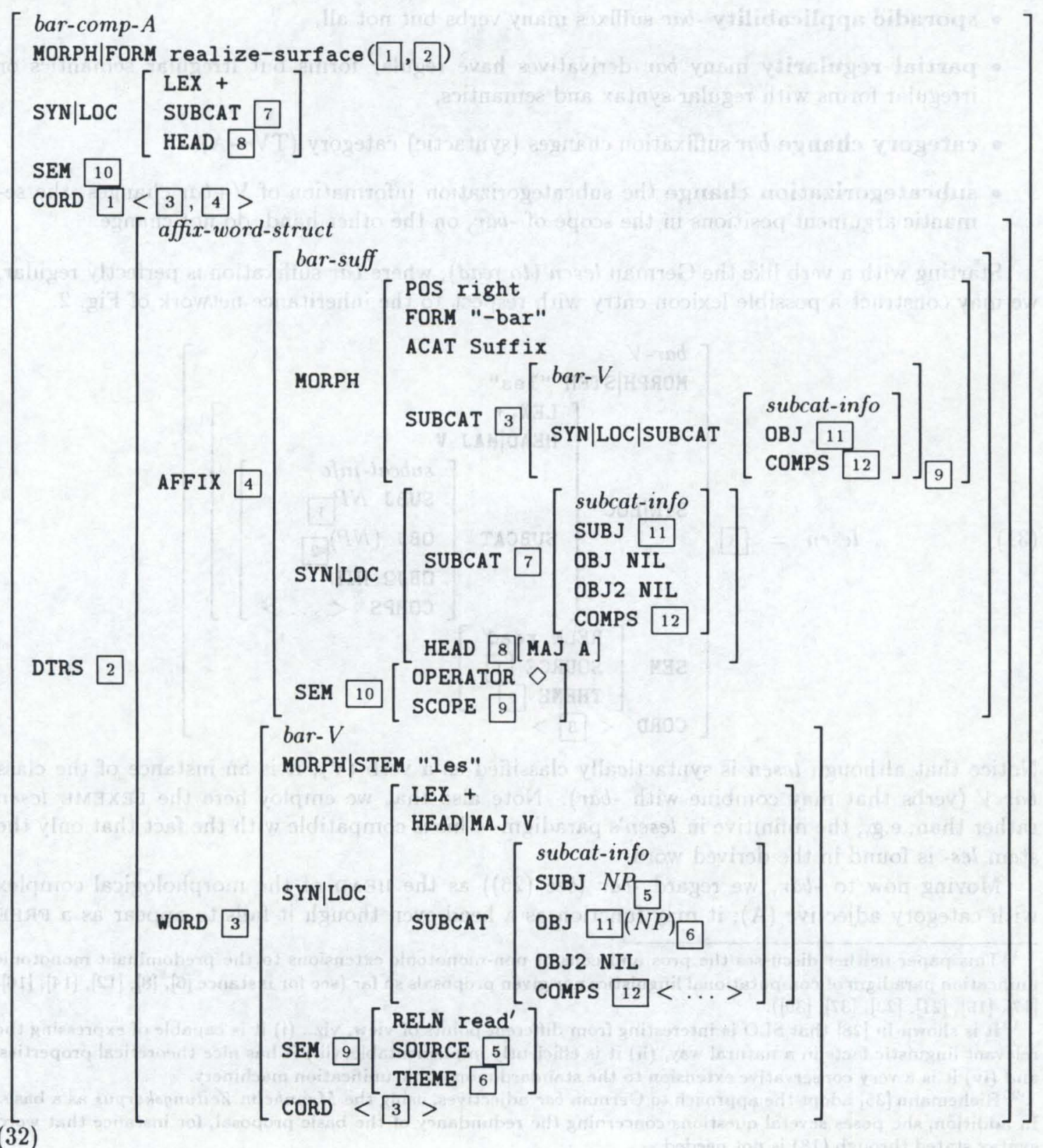


In the same way, we might construct an entry for the complex noun Lesbar+keit (readability) by using the complex adjective les+bar which we had already built-all we have to do further is to specify the feature structure (33) of the suffix -keit, saying that it will subcategorize for objects belonging to the same category lesbar belongs to. The Principles and the Affix-Word Rule then ensure that the feature structure for Lesbarkeit will have the right form. The suffix -keit (33) subcategorizes for a keit adjective keit-A but lesbar (32) is classified as a complex bar adjective bar-comp- $A$. However, this causes no problem when assuming a type hierarchy like the one depicted in Fig. 2, because bar-comp- $A$ is a SUBTYPE of keit- $A$, and -keit is of course allowed to bind a more specific type than keit- $A .^{21}$

$$
\text { keit }=\left[\begin{array}{l}
\text { keit-suff } \\
\text { MORPH }\left[\begin{array}{l}
\text { affix-morphology } \\
\text { FORM "-keit" } \\
\text { SUBCAT keit-A }
\end{array}\right]
\end{array}\right]
$$

Among bar verbs such as lesen, having perfectly regular bar adjectives (i.e., complex adjectives, containing -bar as their head, e.g., lesbar), there are others whose derived adjectives are partially irregular, for example with respect to their form (e.g., sichtbar, kündbar) or their semantics. As an additional complication, some bar adjectives of these verbs are provided with an additional regular, but non-standard reading. Take for instance the German verb essen (to eat):

$$
\text { essen }=\left[\begin{array}{l}
\text { bar- } V \\
\text { MORPH|STEM "ess" } \\
\text { SYN|LOC|SUBCAT }\left[\begin{array}{ll}
\text { SUBJ } & N P \\
\text { OBJ } & (N P) \\
\text { OB } & 2
\end{array}\right] \\
\text { SEM }\left[\begin{array}{l}
\text { RELN eat' } \\
\text { SOURCE } 1 \\
\text { THEME }
\end{array}\right]
\end{array}\right]
$$

The non-standard (semantically regular) reading of eßbar can be built in a regular way by means of the mechanisms described above, taking essen and -bar to form a complex word:

$$
e^{\beta b a r^{\text {non-stand }}}=\left[\begin{array}{l}
\text { bar-comp- } A \\
\text { SEM } 1\left[\begin{array}{l}
\text { OPERATOR } \diamond \\
\text { SCOPE|RELN eat' }
\end{array}\right] \\
\text { DTRS|AFFIX } \mid \text { SEM } 1
\end{array}\right]
$$

The standard reading of eßbar on the other hand is edible (the property of an object which can SAFELY be eaten). Constructing the standard reading (with irregular semantics) for $e \beta b a r$ can be done in our approach in two different ways:

1. We do NOT regard $e ß b a r$ as an instance of the class bar-comp- $A$; instead, $e \beta b a r$ is entered separately as a whole into the lexicon and belongs to a different word class, say ?-A. A treatment of this kind leads us to the question whether the feature structure (36) actually will have a DTRS attribute-since no use need to be made of the structure.

2. The semantics of (35) (the entry which was built regularly) is modified by using a nonmonotonic device to enforce the standard reading. In this case, e $\beta b a r$ (37) still belongs to the class bar-comp- $A$ and all other properties remain the same. The mechanism we want

\footnotetext{
${ }^{21}$ The type keit- $A$ is used to characterize adjectives that can be bound by the suffix -keit but do not necessarily end in the suffix -bar, e.g., the German adjective heilig (holy) which might be bound by -keit to form the word Heiligkeit (holiness).
} 
to employ during the DEFINITION of subregularities and exceptions is a special form of overwriting, so called Single Link Overwriting. ${ }^{22}$

$$
\begin{aligned}
& e \beta b a r^{\text {stand }}=\left[\begin{array}{l}
?-A \wedge \neg b a r-c o m p-A \\
\text { SEM } \mid \text { RELN safely-eat' } \\
\text { DTRS ??? }
\end{array}\right] \\
& e \beta b a r^{\text {stand }}{ }^{\prime}:=e \beta b a r^{\text {non-stand }} \& \text { ! [SEM|RELN safely-eat'] } \\
& =\left[\begin{array}{l}
\text { bar-comp- } A \\
\text { SEM } 1\left[\begin{array}{l}
\text { RELN safely-eat' } \\
\text { SOURCE } \ldots . . . \\
\text { THEME } \ldots . .
\end{array}\right. \\
\text { DTRS|AFFIX|SEM } 2 \\
1 \neq 2
\end{array}\right]
\end{aligned}
$$

The advantage of the second approach is that regular properties of partially regular derivations need not be specified redundantly, as would be the case in the first approach. The use of default specifications thus obtains the same advantages in DERIVATION that Flickinger et al. [20] and Evans and Gazdar [17] have shown in word-class definitions. Defaults, together with the possibility of overwriting defaults in more specific definitions may turn out to be even more important in connection with the analysis of derivational relationships, since these are notoriously irregular in morphological form, syntactic feature assignment, and semantics.

There are also linguistically motivated examples where the stronger Subcategorization Principle $M S C P^{*}$ comes into play and might be prefered, rather than to employ Single Link Overwriting. Take for instance the following example. lesen (to read) and sehen (to see) are closely related transitive verbs $(T V)$, having (nearly) the same morphological, syntactical, and semantical properties. However, it turns out that bar suffixation would predict a wrong morphological form for sehen, viz., sehbar which is out, but only sichtbar is perfect. This and similar facts can be captured by $\operatorname{MSCP}^{*}(28)$ and by assuming an additional morphological subcategorization feature for objects of type part-of-speech, which is typed to affix. To represent these facts we encode -bar (38) as before, saying that it subcategorizes for transitive verbs:

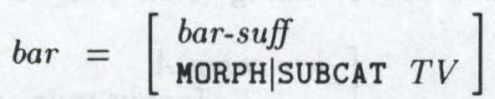

Now, if one wants to state that lesen (39) is a transitive verb $T V$ and regular with respect to the above given subcategorization whereas sehen (40), although belonging to $T V$, fails to form a REGULAR bar derivative, this is easy to encode:

$$
\begin{aligned}
& \text { lesen }=\left[\begin{array}{ll}
T V & \\
\text { MORPH|SUBCAT } & \text { bar-suff }
\end{array}\right] \\
& \text { sehen }=\left[\begin{array}{l}
T V \\
\text { MORPH } \mid \mathrm{SUBCAT} \neg \text { bar-suff }
\end{array}\right]
\end{aligned}
$$

The typed approach to bar suffixation also allows us to prevent ill-formed bar adjectives; e.g., we have to rule out the combination of haben (to have) together with -bar. This is very easy to

\footnotetext{
${ }^{22}$ The term SINGLE LINK OVERWRITING is used to emphasize the fact that overWRITING takes place via a SINGLE INHERITANCE LINK, i.e., defining a new word class using SLO forces us to specify a single class from which we inherit and saying which VALUES we would like to overwrite. Therefore inheritance conflicts (which value to choose?) will never occur because the more specific information always wins (there is always only onE supertype). Note that we are allowed to OVERWRITE COREFERENCE CONSTRAINTS; this was done during the definition of $e \beta b a r$ in (37) because we want to state that the Semantics Principle MSP of course does not hold under this exception. In our special case the syntax is of the following form: new-class := old-class !overwrite-info. SLO will never fail during the definition of a new class under the assumption that the overwrite-info is consistent. However, the closely related notion of SLO UNIFICATION (see [28]) which we would like to apply during processing, might fail-this is in contrast to other proposals which never yield $\perp$, for instance Bouma's Default Unification [6].
} 
achieve under the assumption that haben doesn't belong to the bar verb class bar- $V$, but instead to another class (say ?- $V$ ), thus preventing haben from combining with -bar-therefore hab+bar is disallowed.

$$
\text { haben }=\left[\begin{array}{l}
?-V \wedge \neg b a r-V \\
\cdots \cdots
\end{array}\right]
$$

It is nevertheless possible to construct handhab+bar (manageable) out of handhaben (to handle, to manage), since haben and handhaben are distinct lexemes. By explicitly encoding handhaben as an entry of type bar- $V$, we can move to a legal description of handhabbar.

$$
\begin{gathered}
\text { handhaben }=\left[\begin{array}{c}
\text { bar- } V \\
\ldots .
\end{array}\right] \\
\text { handhabbar }=\left[\begin{array}{l}
\text { bar-comp- } A \\
\text { DTRS }\left[\begin{array}{l}
\text { AFFIX } \mid \text { MORPH } \mid \text { FORM "-bar" } \\
\text { WORD } \mid \text { MORPH } \mid \text { STEM "handhab" }
\end{array}\right]
\end{array}\right]
\end{gathered}
$$

The structure of the class hierarchy (cf. Fig. 2) leads us to a treatment of suffixation (and also of prefixation in general), where the whole process can be described within the framework of inheritance reasoning over feature structure descriptions. On what grounds are we allowed to state such a thesis? At first sight, this statement seems to stand in contrast with the claim made in the beginning, that naive inheritance is not enough. But we do NOT rely on naive inheritance as the ONLY mechanism. So we turn now to an examination of why this is so. We noted earlier that les+bar and Les+bar+keit are legal lexemes because they (and their complex parts) satisfy all principles whose left sides they match (implying that they have to meet the right sides too), and because they are composed out of lexicon entries by means of rules. In doing realistic parsing or generation, we might assume an additional CONTROL MACHINERY outside of the grammar/lexicon, which uses principles and rules to accept or reject, or alternatively, to generate well-formed complex phrases. However because we regard principles as well as rules as types, equation (1) allows us to employ the laws of feature algebras to construct new types (call them PRECATEGORIES), which are subsumed by all principles having a more general left side and by at least one rule (cf. $A W R E P s$ (29) and (30)). Complex words like lesbar on the other hand will then be subsumed by such precategories.

It is now easy to see that the processes described up to now can be represented entirely via inheritance of a sophisticated kind: it is possible to define new legal complex word classes by inheriting from precategories as well as from simple lexical categories (cf. the subtypes of partof-speech in Fig. 2) and by stating additional local constraints for the class in question. Looking at Fig. 2, bar-comp- $A$ is such a complex class. bar-comp- $A$ inherits from keit- $A$ and from the precategory $A W R E P s$, but also enforces idiosyncratic constraints which have to be satisfied by words that are members of this class:

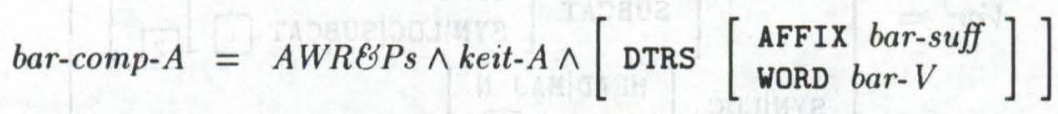

It's very important to constrain AFFIX to bar-suff and WORD to bar- $V$ in order to get the right feature structure for bar-comp- $A$. Since furthermore $A W R \& P s$ (30) is also associated with a feature structure, it's not difficult to construct the prototypical feature structure for bar-comp- $A$ by unifying all the information. But once this is achieved, we may construct an entry for lesbar by instantiating the class bar-comp- $A$ with complement daughter lesen, i.e., WORD must have as its value a feature structure equal to that of the lexeme lesen (31).

$$
\text { lesbar }=b a r-\operatorname{comp}-A \wedge[\text { DTRS } \mid \text { WORD lesen }]
$$

Notice that the feature structure for (45) corresponds to the one of les+bar (32) provided earlier. In entirely the same fashion, we then let create feature structures for new words, e.g., for Les+bar+keit (47). 


$$
\begin{gathered}
\text { keit-comp- } N=A W R E P s \wedge C N \wedge\left[\text { DTRS }\left[\begin{array}{l}
\text { AFFIX keit-suff } \\
\text { WORD keit-A }
\end{array}\right]\right] \\
\text { Lesbarkeit }=\text { keit-comp- } N \wedge[\text { DTRS } \mid \text { WORD lesbar }]
\end{gathered}
$$

\subsection{Prefixation}

What prefixes and suffixes have in common is that they serve as HEADS in our simple headcomplement approach, although they differ in many ways. ${ }^{23}$

This section investigates for further depth the German prefix Vor-, showing how the relevant data about Vor prefixation can be captured properly in our approach presented so far. Some known facts about Vor prefixation:

- sporadic applicability Vor-prefixes many nouns but not all,

- partial regularity many Vor derivatives have regular forms and irregular semantics,

- category constant the syntactic category of the Vor derivative does not change,

- subcategorization constant the subcategorization information of the derived complex word $V o r+\mathrm{N}$ is taken over completely from the complement,

- iterability the prefix Vor-can be applied iteratively.

Let's examine the German noun Version (version) that may combine with Vor- in order to form a complex noun. ${ }^{24}$

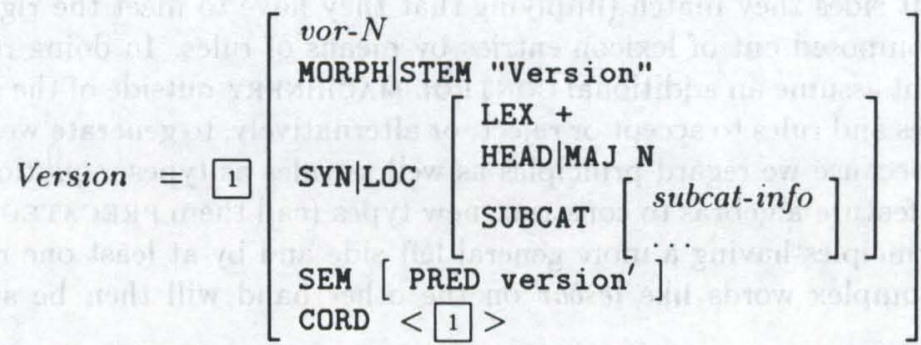

In order to construct Version, Vorversion, Vorvorversion, ..., the prefix Vor- (49) must contain at least the following information. ${ }^{25}$

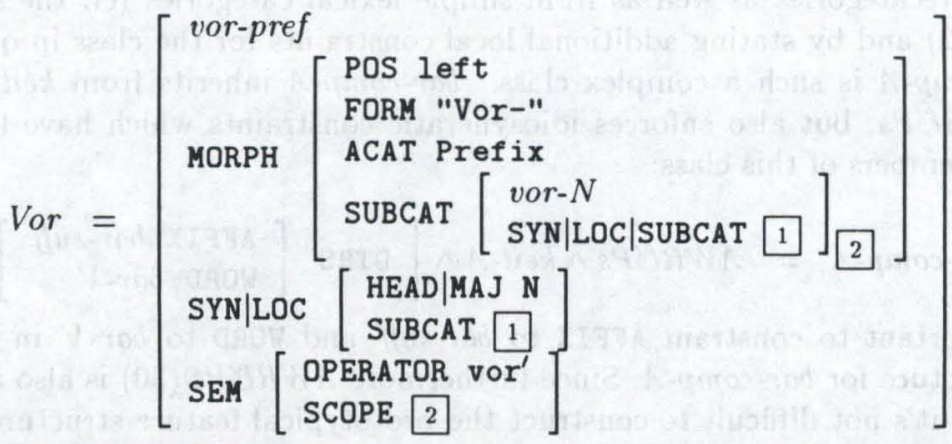

\footnotetext{
${ }^{23}$ One might argue that only suffixes can be regarded as heads and prefixes should be given the status of a modifier (the syntactic category of the compound word is determined by the free word which is the head in this case). However, under this assumption, we have two work with two ID rule schemata, one for prefixes and one for suffixes.

${ }^{24}$ For expository purposes, the semantics of Version is stated as simple as possible, but in general a more complex one must be employed, e.g., the proposal given by Pollard and Sag [34], Ch. 4, for (unsaturated) common nouns.

${ }^{25}$ As we mentioned above, we have taken the prefix Vor-as an example to show how certain phenomena can be handled in our approach. The assumption that Vor-functions semantically as an operator, working on the semantics of the noun is of course not an in-depth analysis and may not be useful in real applications. There are other prefixes like Anti- or $U r$-having similar properties, but their semantics is more complicated.
} 
Note the structure sharing 1 between the sentential subcategorization of the morphologically subcategorized element vor- $N$ and the syntactic subcategorization of Vor-. This is due to the fact that the Subcategorization Principle MSCP coindexes the value of SYN|LOC|SUBCAT in affix and mother. In contrast to -bar, Vor- behaves different to how it contributes to the syntactic subcategorization of the new compound word: the prefix Vor- takes a noun and yields a complex noun which bears the same grammatical relations. By means of the Head Feature Principle $M H F P$, the mother of the morphological phrase will be assigned the same category, the prefix Vor- bears. That's the main reason why RECURSION is possible-the new word Vor+Version (50) will again be classified as a noun and could then combine with a new Vor- to form a more complex word, viz., Vor+Vor+Version in the same way as described before (see analysis tree (51) and the partially depicted feature structure (7) of Vorvorversion). The potential multiple application of Vor- is only allowed because Fig. 3 specifies vor-comp- $N$ as a SUBTYPE of vor- $N$, and Vor-morphologically subcategorizes for objects of type vor- $N$, therefore nouns of the subtype vor-comp- $N$ are especially welcomed.

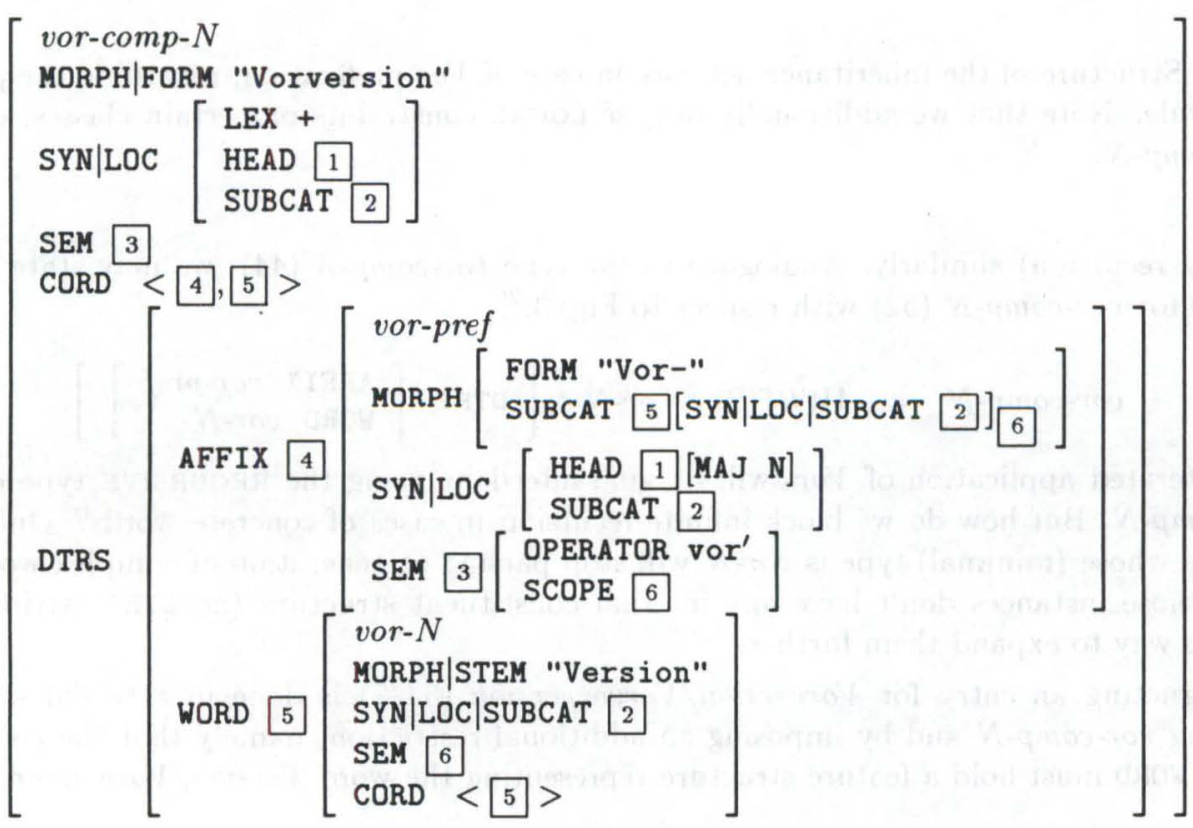

(51)

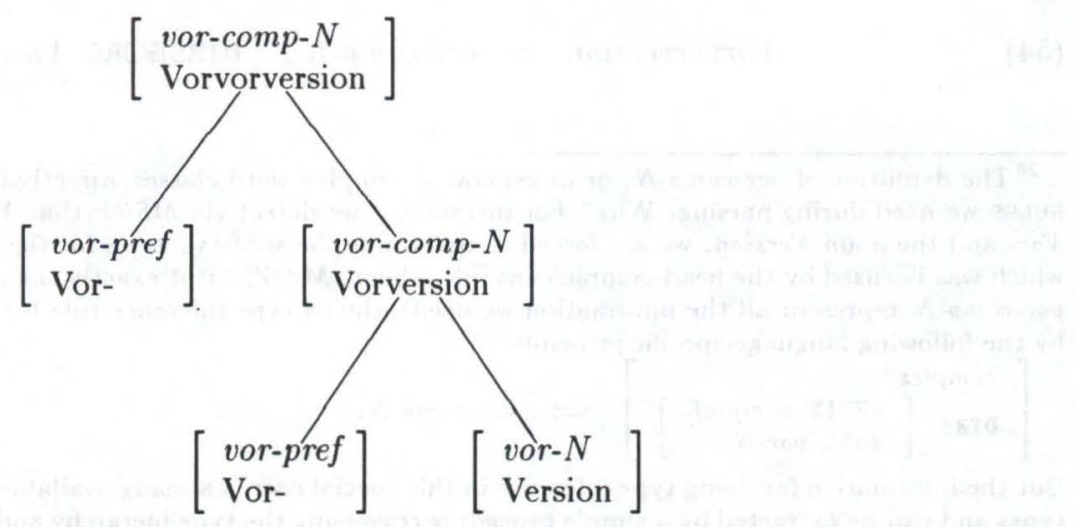

We described bar suffixation above by means of type unification and inheritance over feature terms; once we assume an inheritance network such as Fig. 3, we may analyze Vor prefixation 


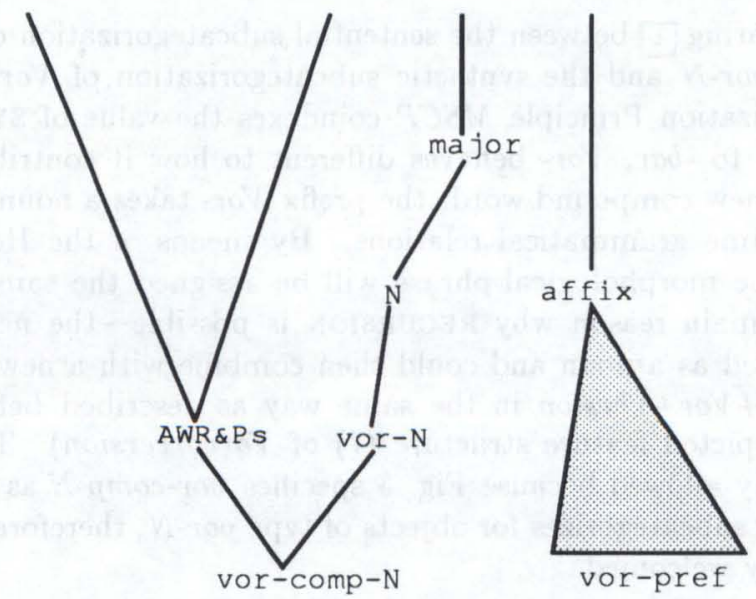

Figure 3: Structure of the inheritance network in case of Vor prefixation, regarding the principles and the rule. Note that we additionally impose LOCAL constraints on certain classes, especially on vor-comp-N.

(including recursion) similarly. Analogous to the type bar-comp-A (44), we may state the right definition for vor-comp-N (52) with respect to Fig. $3 .^{26}$

$$
\text { vor-comp }-N=A W R E P s \wedge \text { vor }-N \wedge\left[\text { DTRS }\left[\begin{array}{l}
\text { AFFIX vor-pref } \\
\text { WORD vor }-N
\end{array}\right]\right]
$$

The iterated application of Vor-will be guaranteed by using the RECuRsive type definition of vor-comp- $N$. But how do we block infinite recursion in cases of concrete words? Only feature structures whose (minimal) type is vor- $N$ will stop parsing or generation of complex word forms, because those instances don't have any internal constituent structure (no DTRS attribute), i.e., there's no way to expand them further.

Constructing an entry for Vorversion/Vorvorversion $(53 / 7)$ is done in a trivial way by instantiating vor-comp- $N$ and by imposing an additional restriction, namely that the complement daughter WORD must hold a feature structure representing the word Version/Vorversion.

$$
\begin{aligned}
\text { Vorversion } & =\text { vor }-\operatorname{comp}-N \wedge[\mathrm{DTRS} \mid \text { WORD Version }] \\
\text { Vorvorversion } & =\text { vor-comp }-N \wedge[\mathrm{DTRS} \mid \text { WORD Vorversion }]
\end{aligned}
$$

\footnotetext{
${ }^{26}$ The definition of vor-comp- $N$, or in general of complex word classes, directly leads us to the TYPE INFERENCE RULES we need during parsing. Why? For instance, if we detect via MSRP that Vorversion consists of the prefix Vor- and the noun Version, we are forced to determine the MINIMAL TYPE for the feature structure of Vorversion which was licensed by the head-complement rule scheme MAWR. But exactly the LOCAL constraints we impose on vor-comp- $N$ represent all the information we need-thus a type inference rule for vor-comp- $N$ might be encoded by the following language-specific principle:

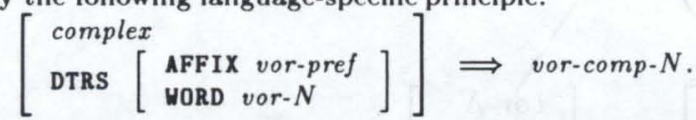

But the information for doing type inference in this special case is already available, viz., in the hierarchy of lexical types and can be extracted by a simple procedure traversing the type hierarchy and yielding a set of type inference rules. However, its a matter of choice whether principles such the one above should be stated explicitly as typed implications in the style of HPSG and processed completely by the underlying unification machinery or should be factored out as context-free rewrite rules, i.e., as phrasal constraints, which can then be applied independently by a special purpose machinery to build up phrase structure trees (see [31] on the advantages of separating phrasal and functional constraints).
} 


\section{The Affix Hierarchy}

It is well known that HPSG was the first linguistic theory of the unification paradigm which incorporated the ideas on STRUCTURED OBJECTS and INHERITANCE, well known from computer science, semantic networks, and terminological knowledge representation systems, to state linguistic knowledge in a distributed way and to structure the lexicon highly modularly, i.e., to capture regularities and to avoid redundant information. Other approaches like LFG or GPSG didn't focus on the lexicon very much - they have either little to say about it or seem to assume a flat lexicon structure.

Because we approach derivation in the same way HPSG handle sentential phenomena, it might be interesting to explore whether the HPSG-style of defining lexical hierarchies can be applied to represent AFFIXES in the same way. ${ }^{27}$ And in fact, the arguments presented by Pollard and Sag for lexical types do hold for affixes as well, so we adopt their notation and cross-classify ALL affixes along certain relevant dimensions at once. For instance, the feature structure of the suffix -bar depicted in (26) is a fully expanded instance of the type bar-suff. However, the IDIOSYNCRATIC PROPERTIES of bar-suff only concern the morphological form, parts of the semantics and the type of the subcategorized object.

Currently FIVE DIMENSIONS contribute to the classification of affixes, but there might be additional generalizations as well:

- POS-is the affix classified as a prefix or as a suffix?

- CAT - does the new word undergo a category change?

- SUBCAT - how does the subcategorization information of the new word look like?

- BIND-which object does the affix morphologically subcategorize for?

- SEM — which form does the semantics of the affix have?

To give an impression how such a hierarchy might look like and how it helps to shorten the definition of a specific affix, we study this mechanism by taking again the suffix -bar (26) as an example. With Fig. 4 in mind, we can express what is idiosyncratic to bar-suff and what must be represented in the types from which bar-suff inherits:

$$
\begin{aligned}
\text { bar-suff }=\text { suffix } & \wedge V \text {-to- } A \wedge \text { drop-subject } \wedge \text { 1-place-operator } \wedge \text { bind- } T V \\
& \wedge\left[\begin{array}{l}
\text { MORPH }\left[\begin{array}{l}
\text { FORM "-bar" } \\
\text { SUBCAT bar-V }
\end{array}\right] \\
\text { SEM } \mid \text { OPERATOR } \diamond
\end{array}\right]
\end{aligned}
$$

(55) classifies bar-suff as a suffix ( $s u f f i x$ ) which triggers a category change from verb to adjective $(V$-to- $A)$ and leads to a set of grammatical relations in the mother with fewer elements than the complement consists of by throwing away the subject (drop-subject); in addition, bar-suff morphologically subcategorizes for a transitive verb (bind-TV) and the semantics is characterized roughly as an operator-scope structure (1-place-operator). Although bar-suff inherits from bind$T V,(55)$ forces MORPH|SUBCAT to be of type bar- $V$, since not all combinations of a transitive verb with the suffix -bar are legal; so we assume that bar verbs are a natural subclass of the transitive verbs, i.e., bar- $V \sqsubset T V$.

Before we go on in representing more affixes in the way above, we have to say a few words about the different dimensions and partitions (types), which can be found in Fig. 4. As a main result, we will show that a feature logic without functions or relations is too WEAK to characterize the SUBCAT dimension.

\footnotetext{
${ }^{27}$ See Pollard and Sag [34] for a motivation and many examples and [43] or [13] for the logic underlying those hierarchies.
} 


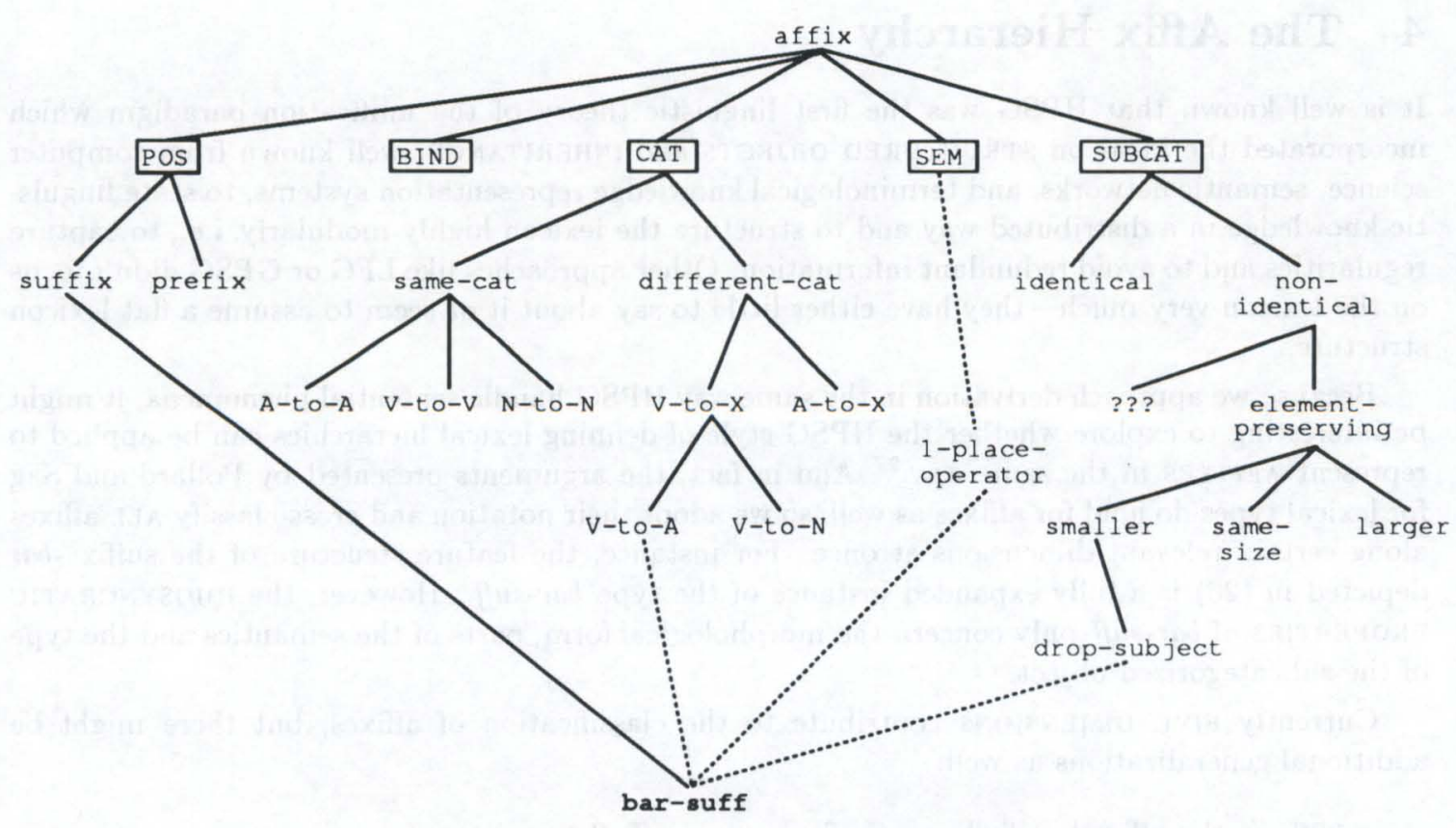

Figure 4: Structure of the cross-classified affix hierarchy with respect to the special suffix type bar-suff. Dashed lines should indicate that there might be additional types which are however not shown in the picture. Note that we omit the whole BIND dimension and also essential parts of the SEM dimension.

\subsection{The POS Dimension}

The POS dimension (see Fig. 4) simply states whether the affix is classified as a prefix (56) or a suffix.

$$
\text { prefix }=\text { affix } \wedge\left[\text { MORPH }\left[\begin{array}{l}
\text { POS left } \\
\text { ACAT Prefix }
\end{array}\right]\right]
$$

\subsection{The CAT Dimension}

The CAT dimension (see Fig. 4) expresses whether the syntactic category of the mother differs from the one of the complement or is identical.

$$
\begin{aligned}
& \text { same-cat }=a f f i x \wedge\left[\begin{array}{l}
\text { MORPH } \mid \text { SUBCAT } \mid \text { SYN } \mid \text { LOC } \mid \text { HEAD } \mid \text { MAJ } 1 \\
\text { SYN } \mid \text { LOC } \mid \text { HEAD } \mid \text { MAJ } 1
\end{array}\right] \\
& \text { different-cat }=\text { affix } \wedge\left[\begin{array}{l}
\text { MORPH } \mid \text { SUBCAT } \mid \text { SYN } \mid \text { LOC } \mid \text { HEAD } \mid \text { MAJ } 1 \\
\text { SYN } 1 \text { LOC } \mid \text { HEAD } \mid \text { MAJ } 2 \\
1 \neq 2
\end{array}\right] \\
& V \text {-to- } X=\text { different-cat } \wedge[\text { MORPH } \mid \text { SUBCAT } \mid \text { SYN } \mid \text { LOC } \mid \text { HEAD } \mid \text { MAJ } \mathbf{V}] \\
& V \text {-to- } A=V \text {-to- } X \wedge[\text { SYN|LOC } \mid \text { HEAD } \mid \text { MAJ A }]
\end{aligned}
$$

\subsection{The SUBCAT Dimension}

Because the subcategorization information of the mother is mainly determined by the complement, the free word, and because of the special nature of the Subcategorization Principle MSCP (27), 
we introduce the SUBCAT dimension (see Fig. 4) to state the relevant generalizations about the sententially subcategorized elements in mother and complement. The first question is whether they are identical $(61)$ or not $(62)$.

$$
\begin{gathered}
\text { identical }=a \text { ffix } \wedge\left[\begin{array}{l}
\text { MORPH } \mid \text { SUBCAT } \mid \text { SYN } \mid \text { LOC } \mid \text { SUBCAT } 1 \\
\text { SYN } \mid \text { LOC } \mid \text { SUBCAT } 1
\end{array}\right] \\
\text { non-identical }=a f f i x \wedge\left[\begin{array}{l}
\text { MORPH } \mid \text { SUBCAT } \mid \text { SYN } \mid \text { LOC } \mid \text { SUBCAT } 1 \\
\text { SYN } \mid \text { LOC } \mid \text { SUBCAT } 2 \\
1 \neq 2
\end{array}\right]
\end{gathered}
$$

If the sentential subcategorization information in mother and complement isn't identical, the question arises whether all subcat elements of the complement can be found in the mother or all elements of the mother occur as parts of the complement (63). However, to formulate such a constraint, we have to use a feature logic that allows us to state functional/relational constraints. Without functions/relations, we cannot express the linguistic generalizations properly, i.e., it turns out that certain type definitions would be too GENERAL, therefore allowing us to predict non-legal descriptions.

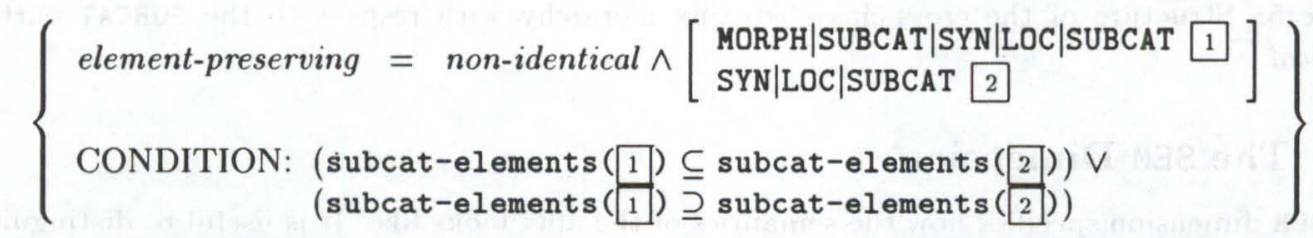

Exactly the CONDITION part of (63) and (64) (and also of other SUBCAT types; see Fig. 4) cannot be represented otherwise. Why? Because the constraint is formulated as a condition that must hold on parts/elements of a set (set inclusion and set membership) and this is outside the expressiveness of (propositional) feature logic. The same is true if we have to formulate that two arbitrary list of unknown length (see the CoMPS attribute in type subcat-info) must have the same elements, i.e., are equal modulo permutations.

$$
\left\{\begin{array}{l}
\text { smaller }=\text { element-preserving } \wedge\left[\begin{array}{l}
\text { MORPH } \mid \text { SUBCAT } \mid \text { SYN } \mid \text { LOC } \mid \text { SUBCAT } 1 \\
\text { SYN } \mid \text { LOC } \mid \text { SUBCAT } 2
\end{array}\right] \\
\text { CONDITION: subcat-elements }(1) \text { D subcat-elements }(2)
\end{array}\right\}
$$

Note that subcat-elements is defined as

$$
\begin{aligned}
& \text { subcat-elements ([SUBJ } 1, \text { OBJ } 2, \text { OBJ2 [3, COMPS } 4]):= \\
& \quad \text { singleton( } 1 \text { ) } \cup \text { singleton }(2]) \cup \text { singleton }(3) \cup \text { ist-to-set }(\square) .
\end{aligned}
$$

drop-subject is the SUBCAT type from which bar-suff directly inherits (cf. (55)). It is responsible that $b a r$, and therefore all complex bar adjectives will get the right sentential subcategorization frame (see subsection on suffixation and cf. the feature structure of bar (26)).

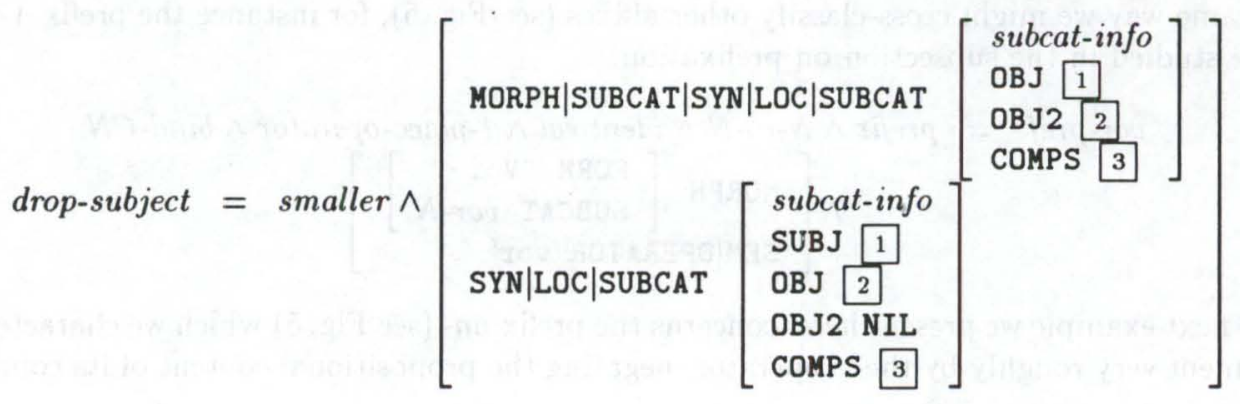




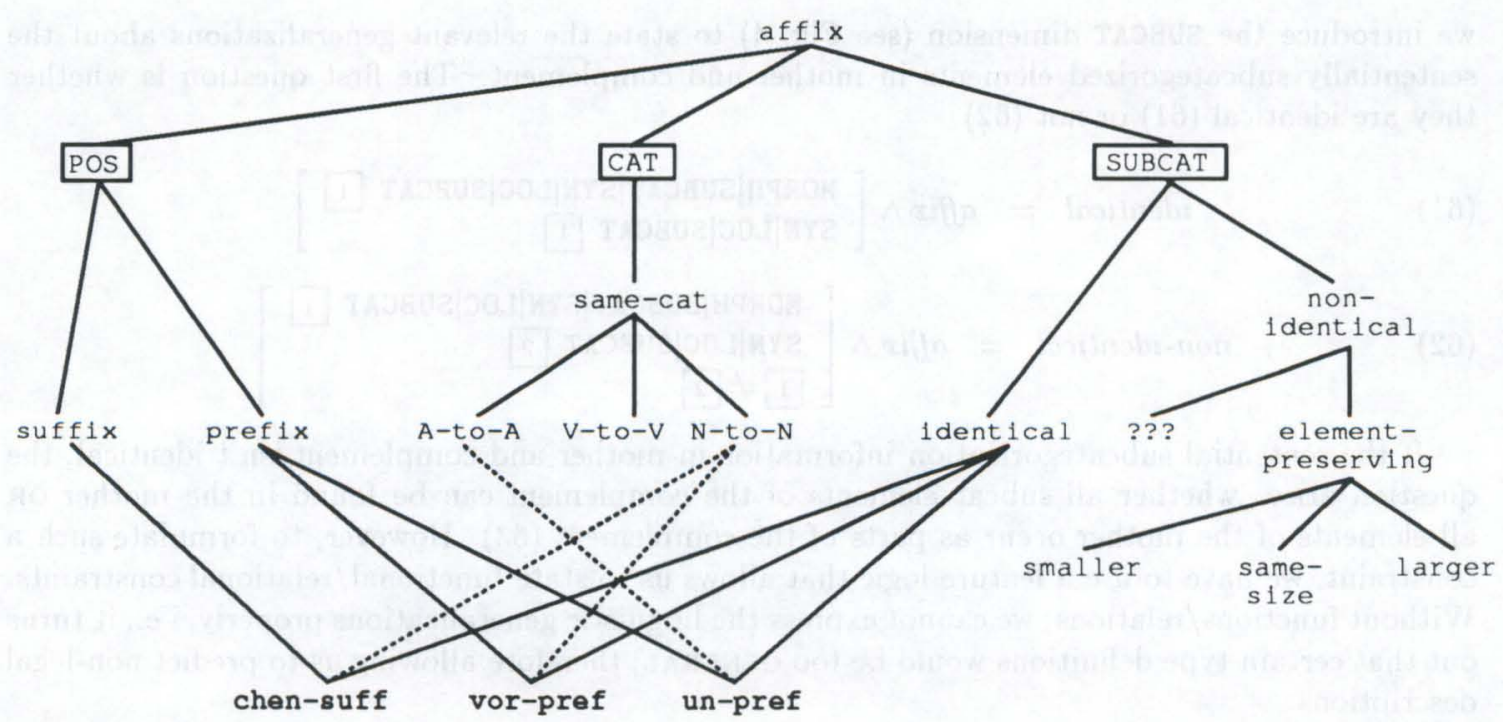

Figure 5: Structure of the cross-classified affix hierarchy with respect to the SUBCAT partition identical.

\subsection{The SEM Dimension}

The SEM dimension specifies how the semantics of the affix looks like. It is useful to distinguish in general between predicate-argument and operator-scope structures. In addition, a differentiation with respect to the arity of predicates/operators does make sense. However, we will give only the definition of the type 1-place-operator which can be found in the definition of bar-suff (55).

$$
\text { 1-place-operator }=\text { affix } \wedge\left[\begin{array}{l}
\text { MORPH|SUBCAT|SEM } 1 \\
\text { SEM }\left[\begin{array}{l}
\text { operator-scope-struct } \\
\text { OPERATOR } \\
\text { SCOPE } 1
\end{array}\right]
\end{array}\right]
$$

\subsection{The BIND Dimension}

The last dimension BIND states which sort of objects an affix morphologically subcategorizes for. It is worth noting that different affixes might share the same BIND dimension, e.g., the German suffixes -chen and -lein morphologically subcategorize for common nouns. The BIND dimension of the type bar-suff (55) above is given by the following feature structure:

$$
\text { bind }-T V=a f f i x \wedge[\text { MORPH } \mid \text { SUBCAT } T V]
$$

\subsection{More Examples}

In the same way we might cross-classify other affixes (see Fig. 5), for instance the prefix Vor- (49), we have studied in the subsection on prefixation.

$$
\begin{aligned}
\text { vor-pref }=\text { prefix } & \wedge N \text {-to- } N \wedge \text { identical } \wedge \text { 1-place-operator } \wedge \text { bind- } C N \\
\wedge & \wedge\left[\begin{array}{l}
\text { MORPH }\left[\begin{array}{l}
\text { FORM "Vor-" } \\
\text { SUBCAT vor- } N
\end{array}\right] \\
\text { SEM } \mid \text { OPERATOR vor' }
\end{array}\right]
\end{aligned}
$$

The next example we present here, concerns the prefix un- (see Fig. 5) which we characterized at the moment very roughly by the $\neg$ operator, negating the propositional content of its complement 
through the value of SCOPE. Again the subcategorization frame of the new word (e.g., the German un+schön-not (very) nice) is equal to the one of the complement, un-subcategorizes for and the syntactic category of the new compound remains the same, viz. adjective (A).

$$
\begin{aligned}
\text { un-pref }=\text { prefix } & \wedge A \text {-to- } A \wedge \text { identical } \wedge \text { 1-place-operator } \wedge \text { bind- } A \\
& \wedge\left[\begin{array}{l}
\text { MORPH } \left.\left[\begin{array}{l}
\text { FORM un-" } \\
\text { SUBCAT un- } A
\end{array}\right]\right] \\
\text { SEM } \mid \text { OPERATOR } \neg
\end{array}\right.
\end{aligned}
$$

Note that bind- $A$ (71) takes care that the MOD feature of the subcategorized adjective is coindexed with the one in the prefix un-. As a consequence of the Head Feature Principle MHFP, the mother also shares the value of the MOD feature.

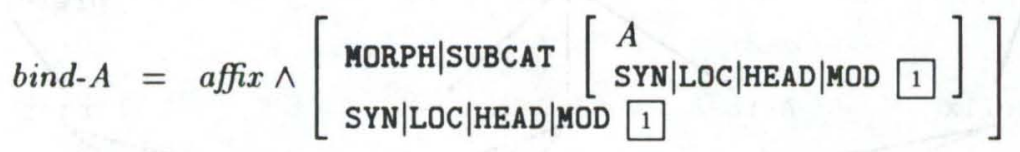

Therefore a fully expanded feature structure for un- is of the following form:

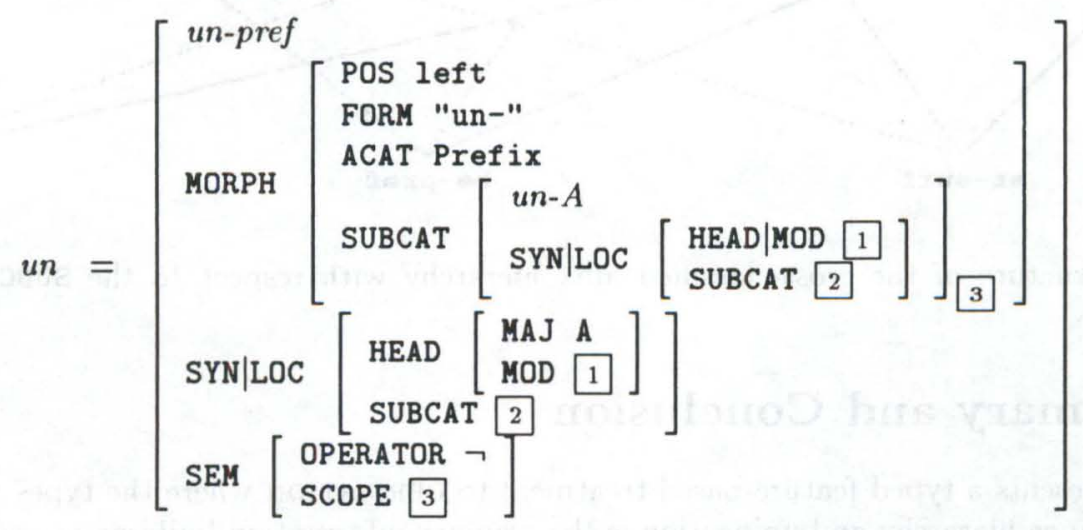

The German diminution operators -chen (see Fig. 5) and -lein are also easy to characterize. ${ }^{28}$

$$
\begin{aligned}
\text { chen-suff }=\text { suffix } & \wedge N \text {-to- } N \wedge \text { identical } \wedge 1 \text {-place-operator } \wedge \text { bind- } N \\
& \wedge\left[\begin{array}{l}
\text { MORPH } \mid \text { FORM "-chen" } \\
\text { SEM } \mid \text { OPERATOR little } \mathbf{e}^{\prime}
\end{array}\right] \\
\text { lein-suff }=\text { suffix } & \wedge N \text {-to- } N \wedge \text { identical } \wedge 1 \text {-place-operator } \wedge \text { bind- } N \\
& \wedge\left[\begin{array}{l}
\text { MORPH } \mid \text { FORM "-lein" } \\
\text { SEM } \mid \text { OPERATOR little' }
\end{array}\right]
\end{aligned}
$$

We stop here in giving more detailed examples, but we would like to remark that many other German affixes can be straightforwardly represented in the way above (see Fig. 6), for instance

- German -er suffix - adjective comparison as derivation,

- German ge- prefix-passive as derivation,

- German $a n-$ and be- prefixes-category change IV $\sim \mathrm{TV}$.

\footnotetext{
${ }^{28}$ It does make sense to propose a direct supertype (say dim-suff) of chen-suff and lein-suff which incorporates all features they share, so that the specifications of chen-suff and lein-suff reduce to chen-suff := dim-suff * [MORPH|FORM "-chen"] and lein-suff := dim-suff \& [MORPH|FORM "-lein"].
} 


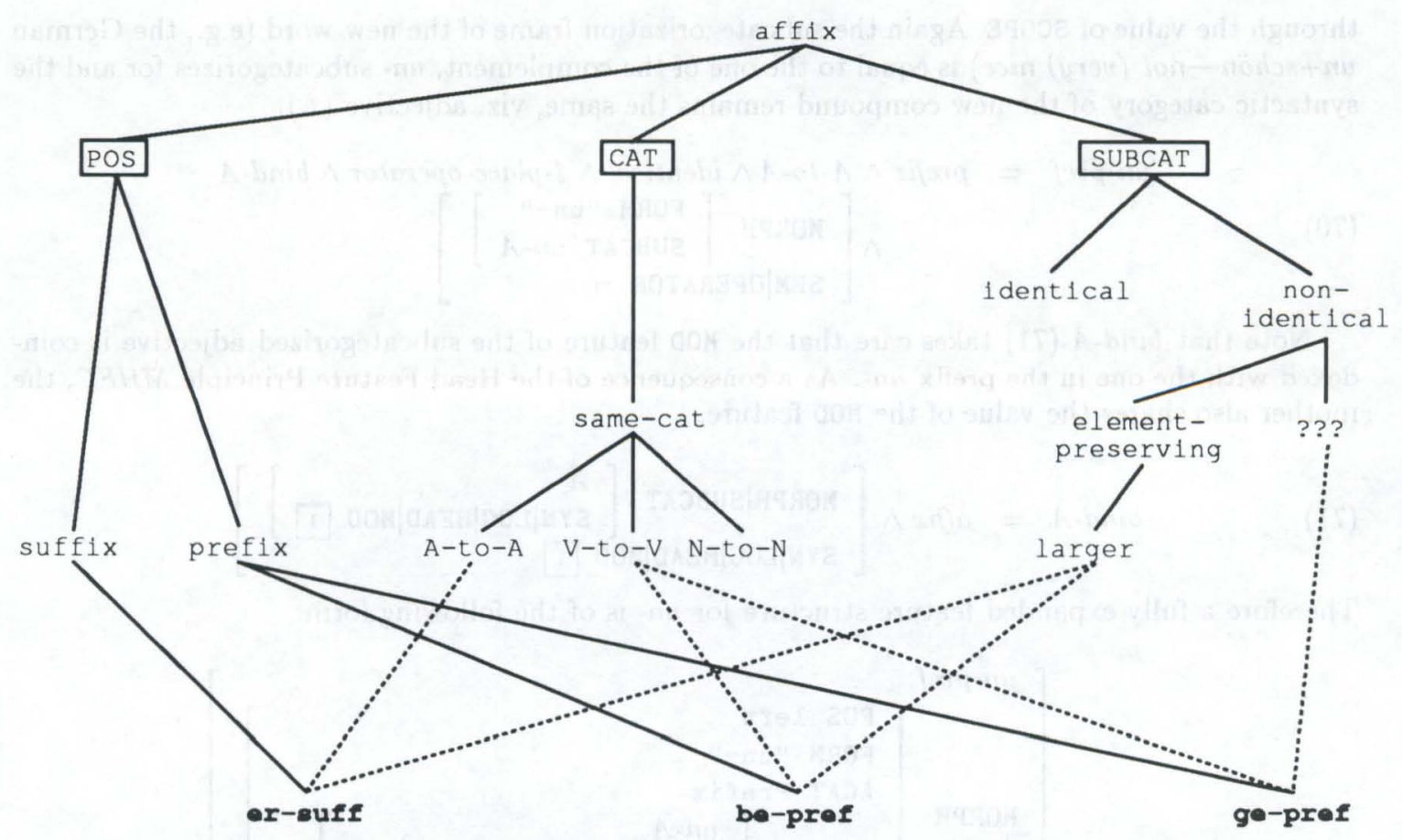

Figure 6: Structure of the cross-classified affix hierarchy with respect to the SUBCAT partition non-identical.

\section{Summary and Conclusion}

The paper presents a typed feature-based treatment to DERIVATION where the types are arranged in an inheritance hierarchy and unification is the primary information-building operation. This is accomplished by proposing more complex feature structures for words (and of course proposing feature structures for affixes) than the ones presented in HPSG and also by proclaiming certain morphological principles and a single rule schema fully in the spirit of HPSG, thus a step further towards a highly lexicalized theory which captures all aspects of linguistic knowledge. Therefore, this approach is an alternative to old-fashioned lexical rules as they are proposed, for instance, in HPSG I, Ch. 8. As a result of this approach, neither a parser nor a generator has to cope with the problem of how to use lexical rules during the processing of sentences - e.g., from a parser's point of view, it makes no difference to work on the word level or on the phrasal/sentential level, in other words, a control machinery doesn't have to switch between the application of traditional lexical rules (word level) and the application of principles and rule schemata (sentential level). But an approach like this one also leads to other benefits as we noted in the introductory chapter.

Another topic we considered extensively, concerns the separation between morphotactics and morphophonemics. This was achieved by proposing a morphological and a sentential subcategorization and by directly recording the constituent order of the parts of a complex word. We argued that it is in fact possible to encode the whole morphotactics in the lexicon by means of the above given approach where the knowledge about morphotactics was made explicit instead of proposing a functional solution. The interface to morphophonemics is of course a functional one, viz., the function realize-surface in the Surface Realization Principle MSRP. This function, however, is set free completely from morphotactics.

One of the main results of this paper concerns the invention of a cross-classified hierarchy of affixes. ${ }^{29}$ We have proposed a distributed representation of many known generalizations about affixes, where the linguistic knowledge is currently factored into five dimensions. A concrete affix

${ }^{29}$ Note that we suggest that sign is now exhaustively partitioned into phrase, word, and affix (see Fig. 1). 
is then composed out of the different dimensions at once by means of unification/inheritance. The nice properties Pollard and Sag have given for the hierarchy of lexical types, can be obtained for free in classifying affixes in the same way.

There are other linguistic areas which we think can be captured by (small extensions of) the treatment we have presented here, viz, COMPOUNDS, IDIOMS, and the whole process of INFLECTION. These three topics will be under investigation in the near future.

\section{References}

[1] Hassan Aït-Kaci. An algebraic semantics approach to the effective resolution of type equations. Theoretical Computer Science, 45:293-351, 1986.

[2] Hassan Aït-Kaci and Roger Nasr. Residuation: A paradigm for integrating logic and functional programming. Technical Report AI-359-86, MCC, Austin, TX, 1986.

[3] Mark Aronoff. Word Formation in Generative Grammar. MIT Press, Cambridge, MA, 1976.

[4] Steven Bird. Finite-state phonology in HPSG. In Proceedings of the 14th International Conference on Computational Linguistics, COLING-92, pages 74-80, 1992.

[5] Robert D. Borsley. Subjects and complements in HPSG. Technical Report CSLI-87-107, Center for the Study of Language and Information, Stanford University, 1987.

[6] Gosse Bouma. Feature structures and nonmonotonicity. Computational Linguistics, 18(2):183-203, 1992.

[7] Joan Bresnan, editor. The Mental Representation of Grammatical Relations. MIT Press, Cambridge, Mass., 1982.

[8] Jonathan Calder. Feature-value logic: Some limits on the role of defaults. In Mike Rosner, C.J. Rupp, and Rod Johnson, editors, Proceedings of the Workshop on Constraint Propagation, Linguistic Description, and Computation, pages 20-32. Instituto Dalle Molle IDSIA, Lugano, 1991.

[9] Luca Cardelli. A semantics of multiple inheritance. Information and Computation, 76:138$164,1988$.

[10] Luca Cardelli and Peter Wegner. On understanding types, data abstraction, and polymorphism. ACM Computing Surveys, 17(4):471-522, 1985.

[11] Bob Carpenter. The Logic of Typed Feature Structures. Tracts in Theoretical Computer Science. Cambridge University Press, Cambridge, 1992.

[12] Bob Carpenter. Skeptical and credulous default unification with applications to templates and inheritance. In Ted Briscoe, Ann Copestake, and Valeria de Paiva, editors, Default Inheritance Within Unification-Based Approaches to the Lexicon. Cambridge University Press, 1992.

[13] Bob Carpenter and Carl Pollard. Inclusion, disjointness and choice: The logic of linguistic classification. In Proceedings of the 29th Annual Meeting of the Association for Computational Linguistics, pages 9-16, 1991.

[14] Ann Copestake. Defaults in the LRL. In Ted Briscoe, Ann Copestake, and Valeria de Paiva, editors, Default Inheritance Within Unification-Based Approaches to the Lexicon. Cambridge University Press, 1992.

[15] Ann Copestake, Valeria de Paiva, and Antonio Sanfilippo. Functionality of the LKB. Draft version, unpublished, University of Cambridge, Computer Laboratory, March 1991. 
[16] Walter Daelemans, Koenraad De Smedt, and Gerald Gazdar. Inheritance in natural language processing. Computational Linguistics, 18(2):205-218, 1992.

[17] Roger Evans and Gerald Gazdar. The DATR papers. Technical Report SCRP 139, School of Cognitive and Computing Sciences, University of Sussex, 1990.

[18] Jens Erik Fenstad, Per-Kristian Halvorsen, Tore Langholm, and Johan van Benthem. Situations, Language and Logic. Reidel, Dordrecht, 1987.

[19] Daniel Flickinger. Lexical Rules in the Hierarchical Lexicon. PhD thesis, Stanford University, 1987.

[20] Daniel Flickinger, Carl Pollard, and Thomas Wasow. Structure-sharing in lexical representation. In Proceedings of the 25th Annual Meeting of the Association for Computational Linguistics, pages 262-267, 1985.

[21] Gerald Gazdar. Linguistic applications of default inheritance mechanisms. In P. Whitelock, M.M. Wood, H.L. Somers, R. Johnson, and P. Bennett, editors, Linguistic Theory and Computer Applications, pages 37-67. Academic Press, London, 1987. Also available as Cognitive Science Research Paper CSRP 070, University of Sussex.

[22] Jack Hoeksema. Categorial Morphology. Garland, New York, 1985.

[23] Ronald M. Kaplan. Three seductions of computational psycholinguistics. In P. Whitelock, M.M. Wood, H.L. Sommers, R. Johnson, and P. Bennett, editors, Linguistic Theory and Computer Applications, pages 149-188. Academic Press, London, 1987.

[24] Lauri Karttunen. D-PATR: A development environment for unification-based grammars. Technical Report CSLI-86-61, Center for the Study of Language and Information, Stanford University, 1986.

[25] Andreas Kathol. Passives without lexical rules. In John Nerbonne, Klaus Netter, and Carl Pollard, editors, HPSG and German. Center for the Study of Language and Information, Stanford, 1993. CSLI Lecture Notes Series.

[26] Kimmo Koskenniemi. A general computational model for word-form recognition and production. In Proceedings of the 10th International Conference on Computational Linguistics, COLING-84, pages 178-181, 1984.

[27] Hans-Ulrich Krieger. Representing and processing finite automata within typed feature formalisms. Technical report, Deutsches Forschungsinstitut für Künstliche Intelligenz, Saarbrücken, Germany, 1993. Forthcoming.

[28] Hans-Ulrich Krieger. Single link overwriting - a conservative non-monotonic extension of unification-based inheritance networks. Technical report, Deutsches Forschungsinstitut für Künstliche Intelligenz, Saarbrücken, Germany, 1993. Forthcoming.

[29] Hans-Ulrich Krieger and John Nerbonne. Feature-based inheritance networks for computational lexicons. In Ted Briscoe, Ann Copestake, and Valeria de Paiva, editors, Default Inheritance Within Unification-Based Approaches to the Lexicon. Cambridge University Press, 1992. A version of this paper is also available as a DFKI Research Report. Also published in Proceedings of the ACQUILEX Workshop on Default Inheritance in the Lexicon, Technical Report No. 238, University of Cambridge, Computer Laboratory, October 1991.

[30] Hans-Ulrich Krieger, John Nerbonne, and Hannes Pirker. Feature-based allomorphy. In Proceedings of the 31st Annual Meeting of the Association for Computational Linguistics, 1993. A version of this paper is also available as a DFKI Research Report. 
[31] John T. Maxwell III and Ronald M. Kaplan. The interface between phrasal and functional constraints. In Mike Rosner, C.J. Rupp, and Rod Johnson, editors, Proceedings of the Workshop on Constraint Propagation, Linguistic Description, and Computation, pages 105-120. Instituto Dalle Molle IDSIA, Lugano, 1991.

[32] Fernando C.N. Pereira and Stuart M. Shieber. Prolog and Natural-Language Analysis. CSLI Lecture Notes, Number 10. Center for the Study of Language and Information, Stanford, 1987.

[33] Carl Pollard. The syntax-semantics interface in a unification-based phrase structure grammar. In Stephan Busemann, Christa Hauenschild, and Carla Umbach, editors, Views of the SyntaxSemantics Interface: Proceedings of the Workshop on "GPSG and Semantics", Technische Universität Berlin, 22-24.Feb 1989, pages 167-184. KIT FAST, Technische Universität Berlin, 1989.

[34] Carl Pollard and Ivan Sag. Information-Based Syntax and Semantics. Vol. I: Fundamentals. CSLI Lecture Notes, Number 13. Center for the Study of Language and Information, Stanford, 1987.

[35] Susanne Riehemann. Word formation in lexical type hierarchies. a case study of bar-adjectives in german. Master's thesis, Eberhard-Karls-Universität Tübingen, Seminar für Sprachwissenschaft, 1993.

[36] Graeme D. Ritchie, Stephen G. Pulman, Alan W. Black, and Graham J. Russell. A computational framework for lexical description. Computational Linguistics, 13(3-4):290-307, 1987.

[37] Graham Russell, John Carroll, and Susan Warwick-Armstrong. Multiple default inheritance in a unification-based lexicon. In Proceedings of the 29th Annual Meeting of the Association for Computational Linguistics, pages 215-221, 1991.

[38] Stuart Shieber, Hans Uszkoreit, Fernando Pereira, Jane Robinson, and Mabry Tyson. The formalism and implementation of PATR-II. In Barbara J. Grosz and Mark E. Stickel, editors, Research on Interactive Acquisition and Use of Knowledge, pages 39-79. AI Center, SRI International, Menlo Park, Cal., 1983.

[39] Stuart M. Shieber. An Introduction to Unification-Based Approaches to Grammar. CSLI Lecture Notes, Number 4. Center for the Study of Language and Information, Stanford, 1986.

[40] Gert Smolka. A feature logic with subsorts. LILOG Report 33, WT LILOG-IBM Germany, Stuttgart, Mai 1988.

[41] Gert Smolka. Feature constraint logic for unification grammars. IWBS Report 93, IWBSIBM Germany, Stuttgart, November 1989.

[42] Gert Smolka. Residuation and guarded rules for constraint-logic programming. Research Report RR-91-13, DFKI, Saarbrücken, 1991.

[43] Gert Smolka and Hassan Aït-Kaci. Inheritance hierarchies: Semantics and unification. Journal of Symbolic Computation, 7:343-370, 1989. 


\section{DFKI Publikationen}

Die folgenden DFKI Veröffentlichungen sowie die aktuelle Liste von allen bisher erschienenen Publikationen können von der oben angegebenen Adresse bezogen werden.

Die Berichte werden, wenn nicht anders gekennzeichnet, kostenlos abgegeben.

\section{DFKI Research Reports}

\section{R R-92-34}

Philipp Hanschke: Terminological Reasoning and Partial Inductive Definitions

23 pages

\section{R R-92-35}

Manfred Meyer:

Using Hierarchical Constraint Satisfaction for

Lathe-Tool Selection in a CIM Environment

18 pages

\section{R R-92-36}

Franz Baader, Philipp Hanschke:

Extensions of Concept Languages for a Mechanical Engineering Application

15 pages

\section{R R-92-37}

Philipp Hanschke: Specifying Role Interaction in Concept Languages

26 pages

R R-92-38

Philipp Hanschke, Manfred Meyer:

An Alternative to H-Subsumption Based on

Terminological Reasoning

9 pages

\section{R R-92-40}

Philipp Hanschke, Knut Hinkelmann: Combining Terminological and Rule-based Reasoning for Abstraction Processes

17 pages

\section{R R-92-41}

Andreas Lux: A Multi-Agent Approach towards Group Scheduling

32 pages

R R -92-42

John Nerbonne:

A Feature-Based Syntax/Semantics Interface 19 pages

\section{DFKI Publications}

The following DFKI publications or the list of all published papers so far can be ordered from the above address.

The reports are distributed free of charge except if otherwise indicated.

\section{R R -92-43}

Christoph Klauck, Jakob Mauss: A Heuristic driven Parser for Attributed Node Labeled Graph Grammars and its Application to Feature Recognition in CIM 17 pages

\section{R R -92-44}

Thomas Rist, Elisabeth André: Incorporating Graphics Design and Realization into the Multimodal Presentation System WIP 15 pages

\section{RR - 92-45}

Elisabeth André, Thomas Rist: The Design of Illustrated Documents as a Planning Task 21 pages

RR-92-46

Elisabeth André, Wolfgang Finkler, Winfried Graf, Thomas Rist, Anne Schauder, Wolfgang Wahlster: WIP: The Automatic Synthesis of Multimodal Presentations

19 pages

\section{R R-92-47}

Frank Bomarius: A Multi-Agent Approach towards Modeling Urban Traffic Scenarios

24 pages

RR - 92-48

Bernhard Nebel, Jana Koehler:

Plan Modifications versus Plan Generation:

A Complexity-Theoretic Perspective

15 pages

\section{R R -92-49}

Christoph Klauck, Ralf Legleitner, Ansgar Bernardi: Heuristic Classification for Automated CAPP 15 pages

R R - 92 - 50

Stephan Busemann:

Generierung natürlicher Sprache 61 Seiten 


\section{RR-92-51}

Hans-Jürgen Bürckert, Werner Nutt:

On Abduction and Answer Generation through

Constrained Resolution

20 pages

RR-92-52

Mathias Bauer, Susanne Biundo, Dietmar Dengler, Jana Koehler, Gabriele Paul: PHI - A Logic-Based Tool for Intelligent Help Systems

14 pages

\section{RR-92-53}

Werner Stephan, Susanne Biundo:

A New Logical Framework for Deductive Planning 15 pages

RR-92-54

Harold Boley: A Direkt Semantic Characterization of RELFUN

30 pages

R R-92-55

John Nerbonne, Joachim Laubsch, Abdel Kader

Diagne, Stephan Oepen: Natural Language

Semantics and Compiler Technology

17 pages

RR-92-56

Armin Laux: Integrating a Modal Logic of

Knowledge into Terminological Logics

34 pages

R R-92-58

Franz Baader, Bernhard Hollunder:

How to Prefer More Specific Defaults in

Terminological Default Logic

31 pages

RR-92-59

Karl Schlechta and David Makinson: On Principles and Problems of Defeasible Inheritance

13 pages

RR-92-60

Karl Schlechta: Defaults, Preorder Semantics and

Circumscription

19 pages

RR-93-02

Wolfgang Wahlster, Elisabeth André, Wolfgang

Finkler, Hans-Jürgen Profitlich, Thomas Rist:

Plan-based Integration of Natural Language and

Graphics Generation

50 pages

RR-93-03

Franz Baader, Berhard Hollunder, Bernhard Nebel, Hans-Jürgen Profitlich, Enrico Franconi:

An Empirical Analysis of Optimization Techniques for Terminological Representation Systems 28 pages
RR-93-04

Christoph Klauck, Johannes Schwagereit:

GGD: Graph Grammar Developer for features in $\mathrm{CAD} / \mathrm{CAM}$

13 pages

RR-93-05

Franz Baader, Klaus Schulz: Combination Techniques and Decision Problems for Disunification 29 pages

RR-93-06

Hans-Jürgen Bürckert, Bernhard Hollunder, Armin Laux: On Skolemization in Constrained Logics 40 pages

RR-93-07

Hans-Jürgen Bürckert, Bernhard Hollunder, Armin

Laux: Concept Logics with Function Symbols

36 pages

RR-93-08

Harold Boley, Philipp Hanschke, Knut Hinkelmann, Manfred Meyer: COLAB: A Hybrid Knowledge Representation and Compilation Laboratory 64 pages

RR-93-09

Philipp Hanschke, Jörg Würtz:

Satisfiability of the Smallest Binary Program

8 Seiten

RR-93-10

Martin Buchheit, Francesco M. Donini, Andrea

Schaerf: Decidable Reasoning in Terminological

Knowledge Representation Systems

35 pages

\section{RR-93-11}

Bernhard Nebel, Hans-Juergen Buerckert:

Reasoning about Temporal Relations:

A Maximal Tractable Subclass of Allen's Interval

Algebra

28 pages

RR-93-12

Pierre Sablayrolles: A Two-Level Semantics for French Expressions of Motion

51 pages

RR-93-13

Franz Baader, Karl Schlechta:

A Semantics for Open Normal Defaults via a

Modified Preferential Approach

25 pages

RR-93-14

Joachim Niehren, Andreas Podelski,Ralf Treinen:

Equational and Membership Constraints for Infinite Trees

33 pages 
RR-93-15

Frank Berger, Thomas Fehrle, Kristof Klöckner, Volker Schölles, Markus A. Thies, Wolfgang

Wahlster: PLUS - Plan-based User Support

Final Project Report

33 pages

RR-93-16

Gert Smolka, Martin Henz, Jörg Würtz: ObjectOriented Concurrent Constraint Programming in $\mathrm{Oz}$ 17 pages

RR-93-18

Klaus Schild: Terminological Cycles and the Propositional $\mu$-Calculus

32 pages

RR-93-20

Franz Baader, Bernhard Hollunder:

Embedding Defaults into Terminological

Knowledge Representation Formalisms

34 pages

RR-93-22

Manfred Meyer, Jörg Müller:

Weak Looking-Ahead and its Application in

Computer-Aided Process Planning

17 pages

R R-93-23

Andreas Dengel, Ottmar Lutzy:

Comparative Study of Connectionist Simulators

20 pages

RR-93-24

Rainer Hoch, Andreas Dengel:

Document Highlighting -

Message Classification in Printed Business Letters

17 pages

RR-93-26

Jörg P. Müller, Markus Pischel: The Agent

Architecture InteRRaP: Concept and Application

99 pages

R R-93-27

Hans-Ulrich Krieger:

Derivation Without Lexical Rules

33 pages

RR-93-28

Hans-Ulrich Krieger, John Nerbonne,

Hannes Pirker: Feature-Based Allomorphy

8 pages

RR-93-33

Bernhard Nebel, Jana Koehler:

Plan Reuse versus Plan Generation: A Theoretical and Empirical Analysis

33 pages

RR-93-34

Wolfgang Wahlster:

Verbmobil Translation of Face-To-Face Dialogs

10 pages
DFKI Technical Memos

TM-91-13

Knut Hinkelmann: Forward Logic Evaluation:

Developing a Compiler from a Partially Evaluated Meta Interpreter

16 pages

TM-91-14

Rainer Bleisinger, Rainer Hoch, Andreas Dengel:

ODA-based modeling for document analysis

14 pages

TM-91-15

Stefan Busemann: Prototypical Concept Formation An Alternative Approach to Knowledge Representation 28 pages

TM-92-01

Lijuan Zhang: Entwurf und Implementierung eines Compilers zur Transformation von

Werkstückrepräsentationen

34 Seiten

TM-92-02

Achim Schupeta: Organizing Communication and Introspection in a Multi-Agent Blocksworld

32 pages

TM-92-03

Mona Singh:

A Cognitiv Analysis of Event Structure

21 pages

TM-92-04

Jürgen Müller, Jörg Müller, Markus Pischel, Ralf Scheidhauer:

On the Representation of Temporal Knowledge

61 pages

TM-92-05

Franz Schmalhofer, Christoph Globig, Jörg Thoben: The refitting of plans by a human expert

10 pages

\section{TM-92-06}

Otto Kühn, Franz Schmalhofer: Hierarchical skeletal plan refinement: Task- and inference structures

14 pages

TM-92-08

Anne Kilger: Realization of Tree Adjoining

Grammars with Unification

27 pages

\section{TM-93-01}

Otto Kühn, Andreas Birk: Reconstructive Integrated Explanation of Lathe Production Plans

20 pages

TM-93-02

Pierre Sablayrolles, Achim Schupeta:,

Conlfict Resolving Negotiation for COoperative

Schedule Management

21 pages 


\section{DFKI Documents}

D-92-15

DFKI Wissenschaftlich-Technischer Jahresbericht 1991

130 Seiten

D-92-16

Judith Engelkamp (Hrsg.): Verzeichnis von Softwarekomponenten für natürlichsprachliche Systeme 189 Seiten

D-92-17

Elisabeth André, Robin Cohen, Winfried Graf,

Bob Kass, Cécile Paris, Wolfgang Wahlster (Eds.): UM92: Third International Workshop on User Modeling, Proceedings

254 pages

Note: This document is available only for a nominal charge of $25 \mathrm{DM}$ (or 15 US-\$).

D-92-18

Klaus Becker: Verfahren der automatisierten Diagnose technischer Systeme 109 Seiten

\section{D-92-19}

Stefan Dittrich, Rainer Hoch: Automatische, Deskriptor-basierte Unterstützung der Dokumentanalyse zur Fokussierung und Klassifizierung von Geschäftsbriefen

107 Seiten

D-92-21

Anne Schauder: Incremental Syntactic Generation of Natural Language with Tree Adjoining Grammars 57 pages

D-92-22

Werner Stein: Indexing Principles for Relational Languages Applied to PROLOG Code Generation 80 pages

\section{D-92-23}

Michael Herfert: Parsen und Generieren der Prologartigen Syntax von RELFUN

51 Seiten

\section{D.92-24}

Jürgen Müller, Donald Steiner (Hrsg.):

Kooperierende Agenten

78 Seiten

\section{D-92-25}

Martin Buchheit: Klassische Kommunikations- und Koordinationsmodelle

31 Seiten

D-92-26

Enno Tolzmann:

Realisierung eines Werkzeugauswahlmoduls mit Hilfe des Constraint-Systems CONTAX 28 Seiten
D-92-27

Martin Harm, Knut Hinkelmann, Thomas Labisch: Integrating Top-down and Bottom-up Reasoning in COLAB

40 pages

D-92-28

Klaus-Peter Gores, Rainer Bleisinger: Ein Modell zur Repräsentation von Nachrichtentypen 56 Seiten

D-93-01

Philipp Hanschke, Thom Frühwirth: Terminological Reasoning with Constraint Handling Rules

12 pages

D-93-02

Gabriele Schmidt, Frank Peters,

Gernod Laufkötter: User Manual of COKAM+

23 pages

D-93-03

Stephan Busemann, Karin Harbusch(Eds.):

DFKI Workshop on Natural Language Systems:

Reusability and Modularity - Proceedings

74 pages

D-93-04

DFKI Wissenschaftlich-Technischer Jahresbericht 1992

194 Seiten

D-93-05

Elisabeth André, Winfried Graf, Jochen Heinsohn, Bernhard Nebel, Hans-Jürgen Profitlich, Thomas

Rist, Wolfgang Wahlster:

PPP: Personalized Plan-Based Presenter

70 pages

D-93-06

Jürgen Müller (Hrsg.):

Beiträge zum Gründungsworkshop der Fachgruppe

Verteilte Künstliche Intelligenz Saarbrücken 29.-

30. April 1993

235 Seiten

Note: This document is available only for a nominal charge of $25 \mathrm{DM}$ (or 15 US-\$).

D-93-07

Klaus-Peter Gores, Rainer Bleisinger:

Ein erwartungsgesteuerter Koordinator zur partiellen Textanalyse

53 Seiten

D-93-08

Thomas Kieninger, Rainer Hoch: Ein Generator mit Anfragesystem für strukturierte Wörterbücher zur Unterstützung von Texterkennung und Textanalyse 125 Seiten

D-93-09

Hans-Ulrich Krieger, Ulrich Schäfer:

TDL ExtraLight User's Guide

35 pages 


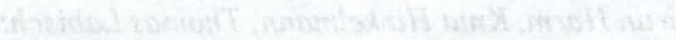

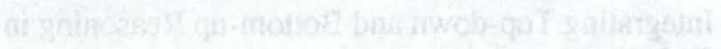

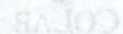

$$
206-2=0-97
$$

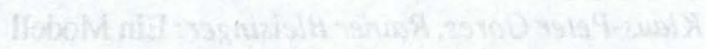

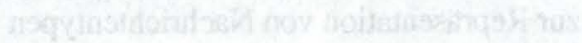

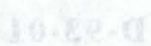

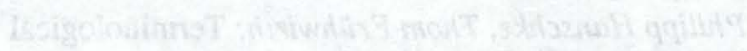

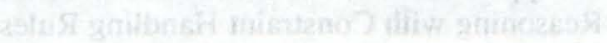

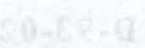

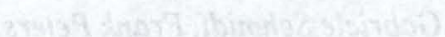

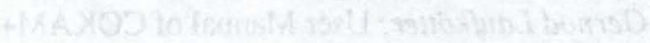

$$
204+4 \text { हल }
$$

$20+24-17$

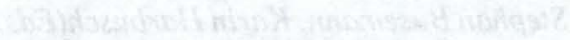

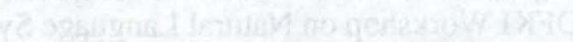

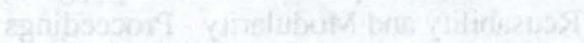
angen the

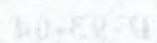

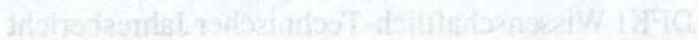

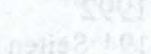

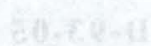

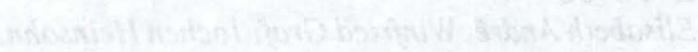

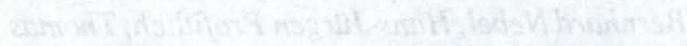

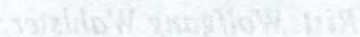

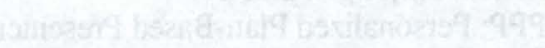

$x+1+59+4$

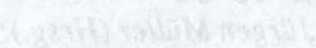

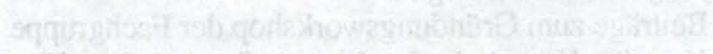

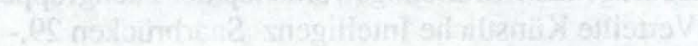

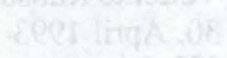

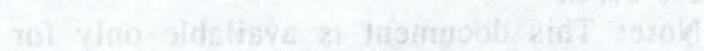

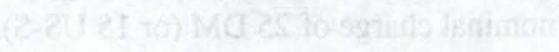

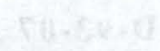

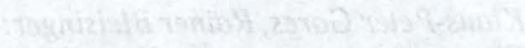

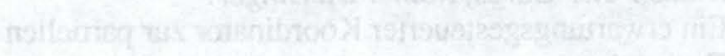

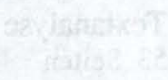
$80+240$

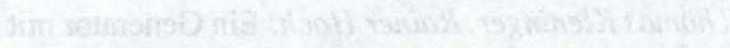

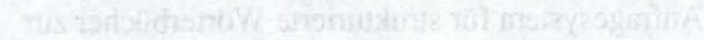

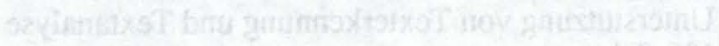

actise 201

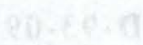

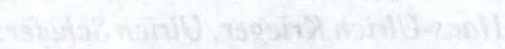

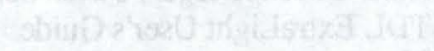

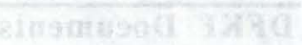
$21+09+4$

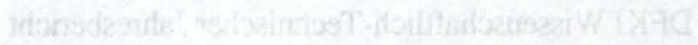
foritis

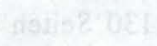

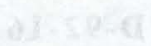

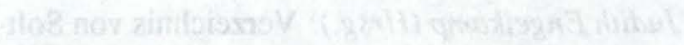

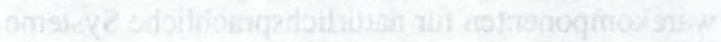

C. 0.4

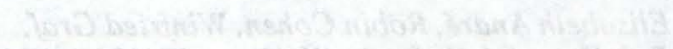

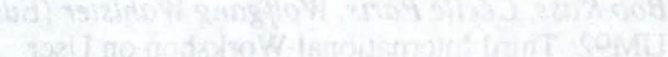

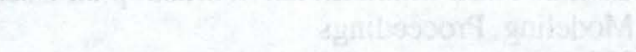

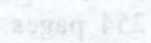

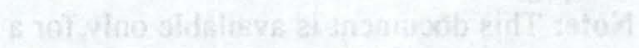

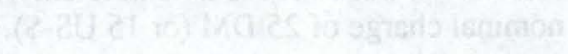
$30 x+2-3$

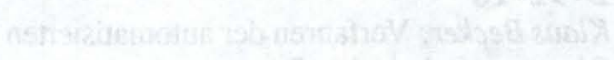

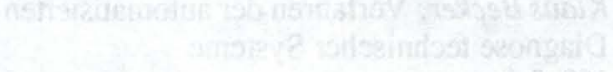
natas 2801

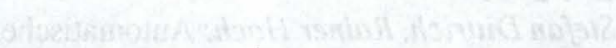

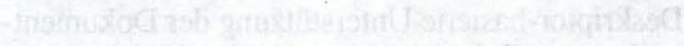

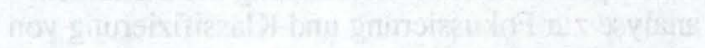

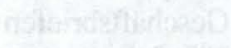

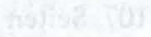

$10-3060$

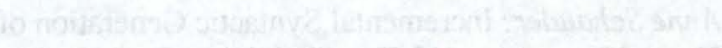

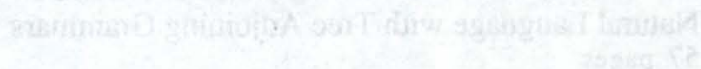

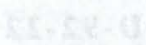

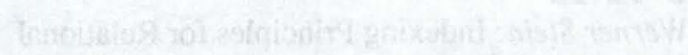

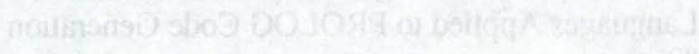

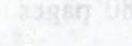
$4+2-5=0-17$

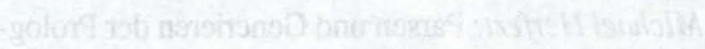

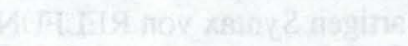

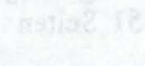

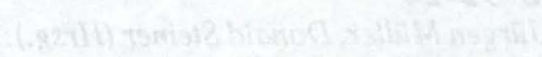

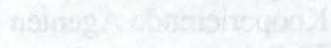

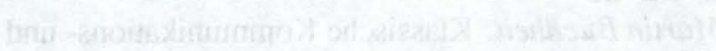

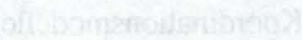
$x^{2}+x^{2}=0$

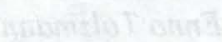

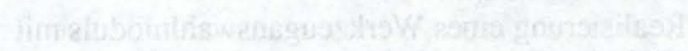

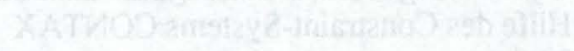



Derivation Without Lexical Rules

RR-93-27

Hans-Ulrich Krieger

\author{
Research Report
}

